\title{
Review
}

Laurens D. Eeftinck Schattenkerk*, Gijsbert D. Musters, David J. Nijssen, Wouter J. de Jonge, Ralph de Vries, L.W. Ernest van Heurn and Joep P.M. Derikx

\section{The incidence of different forms of ileus following surgery for abdominal birth defects in infants: a systematic review with a meta-analysis method}

https://doi.org/10.1515/iss-2020-0042

Received December 7, 2020; accepted April 6, 2021;

published online August 17, 2021

\section{Abstract}

Objectives: Ileus following surgery can arise in different forms namely as paralytic ileus, adhesive small bowel obstruction or as anastomotic stenosis. The incidences of these different forms of ileus are not well known after abdominal birth defect surgery in infants. Therefore, this review aims to estimate the incidence in general between abdominal birth defects.

Content: Studies reporting on paralytic ileus, adhesive small bowel obstruction or anastomotic stenosis were considered eligible. PubMed and Embase were searched and risk of bias was assessed. Primary outcome was the incidence of complications. A meta-analysis was

*Corresponding author: Laurens D. Eeftinck Schattenkerk (LES), MD, Department of Paediatric Surgery, Emma Children's Hospital, Amsterdam University Medical Center, University of Amsterdam and Vrije Universiteit Amsterdam, Meibergdreef 9, 1005 AZ, Amsterdam, Netherlands; and Tytgat Institute for Liver and Intestinal Research, Amsterdam UMC, University of Amsterdam, Amsterdam, Netherlands, Phone: +31 (0)20 5669111 ,

E-mail: l.d.eeftinckschattenkerk@amsterdamumc.nl

https://orcid.org/0000-0002-1069-7062

Gijsbert D. Musters and David J. Nijssen, Department of Paediatric Surgery, Emma Children's Hospital, Amsterdam UMC, University of Amsterdam and Vrije Universiteit Amsterdam, Amsterdam, Netherlands

Wouter J. de Jonge, Tytgat Institute for Liver and Intestinal Research, Amsterdam UMC, University of Amsterdam, Amsterdam, Netherlands; and Department of General, Visceral-, Thoracic and Vascular Surgery, University Hospital Bonn, Bonn, Germany

Ralph de Vries, Medical Library, Vrije Universiteit, Amsterdam, Netherlands

L.W. Ernest van Heurn and Joep P.M. Derikx, Department of Paediatric Surgery, Emma Children's Hospital, Amsterdam UMC, University of Amsterdam and Vrije Universiteit Amsterdam, Amsterdam, Netherlands; and Tytgat Institute for Liver and Intestinal Research, Amsterdam UMC, University of Amsterdam, Amsterdam, Netherlands performed to pool the reported incidences in total and per birth defect separately.

Summary: This study represents a total of 11,617 patients described in 152 studies of which 86 (56\%) had a follow-up of at least half a year. Pooled proportions were calculated as follows; paralytic ileus: $0.07\left(95 \%-\mathrm{CI}, 0.05-0.11 ; I^{2}=71 \%, \mathrm{p} \leq 0.01\right)$ ranging from 0.14 (95\% CI: $0.08-0.23)$ in gastroschisis to 0.05 (95\%-CI: 0.02-0.13) in omphalocele. Adhesive small bowel obstruction: 0.06 (95\%-CI: $0.05-0.07 ; I^{2}=74 \%, \mathrm{p} \leq 0.01$ ) ranging from 0.11 (95\% CI: 0.06-0.19) in malrotation to 0.03 (95\% CI: 0.02-0.06) in anorectal malformations. Anastomotic stenosis after a month 0.04 (95\%-CI: 0.03-0.06; $I^{2}=59 \%, \mathrm{p}=0.30$ ) ranging from 0.08 (95\% CI: 0.04-0.14) in gastroschisis to 0.02 (95\% CI: 0.01-0.04) in duodenal obstruction. Anastomotic stenosis within a month $0.03\left(95 \%\right.$-CI $0.01-0.10 ; I^{2}=81 \%$, $\mathrm{p}=0.02$ ) was reviewed withoutseparateanalysis perbirth defect. Outlook: This review is the first to aggregate the known literature in order approximate the incidence of different forms of ileus for different abdominal birth defects. We showed these complications are common and the distribution varies between birth defects. Knowing which birth defects are most at risk can aid clinicians in taking prompt action, such as nasogastric tube placement, when an ileus is suspected. Future research should focus on the identification of risk factors and preventative measures. The incidences provided by this review can be used in those studies as a starting point for sample size calculations.

Keywords: abdominal birth defect; adhesive small-bowel obstruction; anastomotic stenosis; ileus; infant; paediatric surgery.

\section{Introduction}

Ileus following surgery, consisting of both paralytic and mechanical causes, is a frequent complication after abdominal surgery, leading to increased morbidity, mortality, medical costs, and increased length of hospital stay [1-4]. It is a clinical diagnosis which is characterized by intolerance to 
oral feeds, vomiting, abdominal distention, and the absence of flatus or stool.

Ileus following surgery can arise in different forms which depend on the definition used. Common causes are paralytic ileus, adhesive small bowel obstruction (SBO) and anastomotic stenosis. Paralytic ileus is a transient form of ileus which arises shortly after the operation in a response to surgical stress and is based on temporarily intestinal paralysis [5]. Anastomotic stenosis and SBO are mechanical forms of ileus that present later after surgery and could lead to reoperation.

Since abdominal birth defects are rare, the incidence of these forms of ileus are not well known. Yet, knowing the incidences would provide context for clinical decision making as well as a starting point for future research into preventative measures. Therefore, the objective of this review is to systematically aggregate the available data on the incidence of different forms of ileus following surgery for birth defects in infants.

\section{Methods}

Studies were selected according to the criteria outlined below based on the PRISMA Guidelines [6]. Our protocol has been registered with the International Prospective Register of Systematic Reviews (PROSPERO) on 7 March 2019 (registration number: CRD42019119268).

\section{Participants}

All studies reporting on any form of ileus following surgery for birth defects as primary of secondary endpoint were considered eligible. Only articles that described infants (sthree years) and specifically named the different forms of ileus were included. Animal studies, in vitro studies, nonEnglish or non-Dutch articles, congress abstracts and studies with less than 10 cases were excluded.

\section{Search strategy}

The electronic databases of the National Institutes of Health PubMed and EMBASE were systematically searched in February 2020 using both simple search terms as well as hierarchical family forms (e.g. MESH). The search strategy was designed together with a medical information specialist (RV). It combined four groups of search terms and their equivalents [1]: terms related to the age group of the patients at the moment of surgery (e.g. infantile patients) [2]; terms related to the location of surgery (e.g. abdominal surgery) [3]; terms related to congenital abdominal anomalies (e.g. gastroschisis) [4]; terms related to post-operative complications (e.g. adhesive ileus). Mesh and search terms used in Pubmed are included in Appendix 1.

\section{Primary and secondary outcomes}

The primary endpoint was the pooled percentage of the three forms of ileus. We separately reviewed anastomotic stenosis within a month and after one month. Secondary endpoint was the pooled percentage per birth defect.

Terms included in paralytic ileus are: ileus (not related to anastomotic stricture) and post-operative ileus. Terms that only implied feeding problems without specifying the reason were not included. Terms included in adhesive small bowel obstruction are: intestinal obstruction, small bowel obstruction, adhesive ileus and stricture (not related to anastomosis). No additional terms or definitions were used for anastomotic stenosis; each article that specifically stated anastomotic stenosis was included. The early anastomotic stenosis, occurring within one month, were excluded for the analysis into late onset anastomotic stenosis.

For each complication separately, a Forest plot was created containing the estimated overall pooled proportion and the corresponding 95\%-CIs. In each Forest plot, we also reported the pooled proportion and CIs per disease if [1]; at least three studies reported the specific complication in the disease or [2] if the total number of patients with the disease was $\geq 100$ combined [3]; there was at least one event of a complication present in all studies on a specific disease combined. Birth defects that did not meet these criteria were present in the overall pooled proportion and reported as residuals. Follow-up was reported when relevant.

For all studies with multiple arms, data of both trialarms were combined. If only one arm matched the inclusion criteria, the appropriate arm was used. Additionally extracted parameters were: author, country of conduct, year of publication, journal, study design, duration of follow-up, number of participants and type of birth defect.

\section{Data extraction}

Titles and abstracts were screened to exclude nonrelated publications. Screening was done by two independent authors (LES, DN) using Rayyan. Disagreements were resolved by discussion between the two reviewers. If no consensus could be reached, a third specialist author was consulted (JD, GM). Then, the full texts of the remaining articles were read to determine eligibility for inclusion (LES, DN). If the full text was not found the authors were 
contacted. The reference lists of the included articles were cross checked to find additional articles.

\section{Validity and eligibility assessment}

All included articles were assessed for the methodological quality and risk of bias. For cohort studies the Newcastle Ottawa quality assessment scale was used [7]. In randomized controlled trials this was done using the
Jadad scoring system [8]. The assessment was done by LES and DN separately.

\section{Data synthesis}

For each complication and each disease in a study, a weighted average of the logit proportions was determined by the use of the generic inverse variance method. The logit proportions were back transformed to the summary estimate

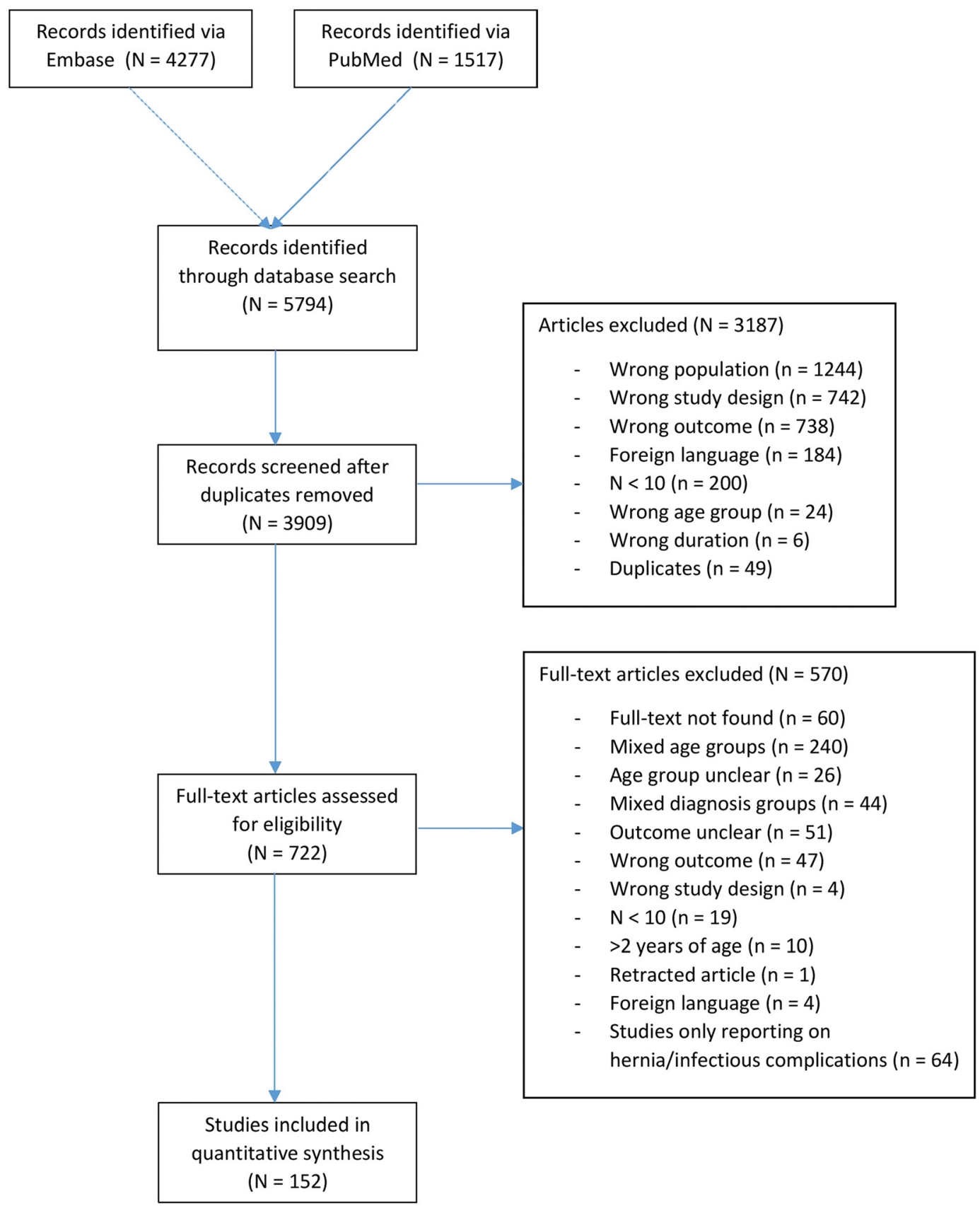

Figure 1: Flow-chart article selection. 
and 95\%-CIs were obtained in a summary proportion representing the pooled proportion of the form of ileus. Heterogeneity was assessed using the $I^{2}$ and $\chi^{2}$ statistics. Analyses were performed with the use of R-studio version 3.6.1 (package "meta" (Schwarzer, 2007) and "metaprop" (Viachtbauer, 2010)). The random-effects model was used for interpretation. Heterogeneity was deemed significant if the pooled data's $\mathrm{p}$ value was $<0.05$ or $\chi^{2}$ statistics were $\geq 75$. Heterogeneity was interpreted as small $\left(I^{2} \leq 0.25\right)$, medium $\left(I^{2}=0.25-0.50\right)$ or strong $\left(I^{2} \geq 0.50\right)$, according to Higgins [9].

\section{Results}

\section{Study characteristics}

In total, 5,784 records were identified. After automated removal of duplicates, 3,909 records were left for title and abstract screening. Of the 3,909 records, 722 were included and assessed for full text. Following full text evaluation 152 studies were included for quantitative analysis (Figure 1). Of the 152 studies, 118 were retrospective cohort studies, 25 were prospective cohort studies, four were retrospective multicentre cohort studies, two were retrospective matched case-control studies, two were randomized controlled trials and one was a combined study of a prospective and retrospective cohort. Studies were conducted in 31 different countries. Asian countries were most prominent with 58 studies, European studies represented 41, North- \& South-America represented 38, Africa represented 9, The Middle-East 4 and Oceania represented 2. Of the 152 studies, 86 (57\%) reported a follow-up of at least half a year.

This systematic review and meta-analysis represent 11,617 patients described in 152 studies presented in Table 1 [10-161]. Among these patients, the congenital conditions were divided as follows: Hirschsprung's disease $(n=4,341$, $37 \%)$; gastroschisis $(\mathrm{n}=1,558,13 \%)$; duodenal obstruction $(\mathrm{n}=1,068,9 \%)$; anorectal malformations $(\mathrm{n}=1,047,9 \%)$; small intestinal atresia $(\mathrm{n}=794,7 \%)$; congenital diaphragmatic hernia $(\mathrm{n}=778,7 \%)$; biliary atresia $(\mathrm{n}=681,6 \%)$; malrotation $(\mathrm{n}=608,5 \%)$; omphalocele $(\mathrm{n}=464,4 \%)$; choledochal cyst $(\mathrm{n}=148,1 \%)$; meconium ileus $(\mathrm{n}=54,>1 \%)$; Meckel's diverticula $(\mathrm{n}=46,>1 \%)$; colonic atresia $(\mathrm{n}=30,>1 \%)$.

Risk of bias was assessed and is shown in Table 2. Most studies included reported fair quality on the NOS which was also the case for the two RCTs using the Jadad score. The mean scores on the NOS of articles describing paralytic ileus and early anastomotic stenosis was slightly lower (5, 5) compared to articles describing adhesive small bowel obstruction and late onset anastomotic stenosis [6].

\section{Paralytic ileus}

In total, 22 studies reported on paralytic ileus and entailed 1,332 patients and 120 events of paralytic ileus [35, 42, 46, 48, 49, 51, 61, 65, 81, 82, 96, 98, 100, 103, 109, 112, 114, 115, $132,151,153,155]$. The pooled proportion of total paralytic ileus was 0.07 (95\%-CI: 0.05-0.11; $I^{2}=71 \%, \mathrm{p} \leq 0.01$ ).

Separate pooled proportions were calculated for the following conditions: Hirschsprung's disease 0.07 (95\%-CI: $\left.0.02-0.24 ; \mathrm{n}=45 / 426 ; I^{2}=91 \% ; \mathrm{p} \leq 0.01\right)$; small intestinal atresia 0.05 (95\%-CI: 0.03-0.09; $\mathrm{n}=16 / 314 ; I^{2}=18 \% ; \mathrm{p}=0.25$ ); gastroschisis 0.14 (95\%-CI: $0.08-0.23 ; \mathrm{n}=30 / 228 ; I^{2}=52 \%$; $\mathrm{p}=0.03$ ); biliary atresia 0.05 (95\%-CI: $0.02-0.11 ; \mathrm{n}=5 / 103$; $I^{2}=0 \% ; \mathrm{p}=0.45$ ); omphalocele 0.05 (95\%-CI: $0.02-0.13 ; \mathrm{n}=5 /$ 96; $\left.I^{2}=8 \% ; \mathrm{p}=0.27\right)$; anorectal malformations 0.06 (95\%-CI: $\left.0.03-0.15 ; \mathrm{n}=5 / 77 ; I^{2}=0 \% ; \mathrm{p}=0.83\right)$. Duodenal obstruction $(\mathrm{n}=58)$ and congenital diaphragmatic hernia $(\mathrm{n}=30)$ are included in the overall proportion but did not meet the criteria for separate statistical analysis (Figure 2).

\section{Adhesive small bowel obstruction (SBO)}

In total, 99 studies reported on small bowel obstruction entailing 8,470 patients and 572 events of SBO all anomalies combined $[10,11,14-19,21,24,26,29-31,33,34,37-41,43$, $45,47,49,51-54,56-60,62,63,67,70-74,79-81,85-89$, 92-94, 96, 97, 99-101, 103, 105, 106, 109-111, 113, 115-121, 123-126, 128, 131, 132, 134, 136-150, 152, 154, 156-160]. Length of follow up was at least half a year in $56(57 \%)$ of the studies.

The pooled proportion of total SBO was 0.06 (95\%-CI: $\left.0.05-0.07 ; I^{2}=74 \%, \mathrm{p} \leq 0.01\right)$. Separate proportions were calculated for the following conditions: Hirschsprung's disease 0.05 (95\%-CI: $0.03-0.07 ; \mathrm{n}=174 / 3,044 ; I^{2}=77 \%$; $\mathrm{p} \leq 0.01$ ); gastroschisis 0.09 (95\%-CI: $0.06-0.14 ; \mathrm{n}=130$ / 1,$\left.147 ; I^{2}=75 \% ; \mathrm{p} \leq 0.01\right)$; congenital diaphragmatic hernia 0.08 (95\%-CI: $0.06-0.11 ; \mathrm{n}=60 / 736 ; I^{2}=35 \% ; \mathrm{p}=0.09$ ); duodenal obstruction 0.04 (95\%-CI: 0.02-0.08; $\mathrm{n}=28 / 728$; $I^{2}=61 \%$; $\left.\mathrm{p}=0.01\right)$; small intestinal atresia 0.09 (95\%-CI: 0.05$\left.0.14 ; \mathrm{n}=60 / 690 ; I^{2}=74 \% ; \mathrm{p} \leq 0.01\right)$; biliary atresia $0.03(95 \%$ CI: $\left.0.02-0.05 ; \mathrm{n}=19 / 543 ; I^{2}=0 \% ; \mathrm{p}=0.48\right)$; malrotation 0.11 (95\%-CI: 0.06-0.19; $\mathrm{n}=46 / 492 ; I^{2}=73 \% ; \mathrm{p} \leq 0.01$ ); anorectal malformations 0.03 (95\%-CI: 0.02-0.06; $\mathrm{n}=17 / 476 ; I^{2}=37 \%$; $\mathrm{p}=0.54$ ); omphalocele 0.04 (95\%-CI: 0.02-0.24; $\mathrm{n}=21 / 357$; $I^{2}=51 \%$; $\mathrm{p}=0.31$ ); choledochal cyst 0.03 (95\%-CI: 0.01-0.08; $\left.\mathrm{n}=5 / 143 ; I^{2}=0 \% ; \mathrm{p}=0.48\right)$. Meconium ileus ( $\left.\mathrm{n}=54\right)$, Meckel's diverticula ( $n=46)$ and colonic atresia $(n=14)$ are included in the overall proportion but did not meet the criteria for separate statistical analysis (Figure 3). 


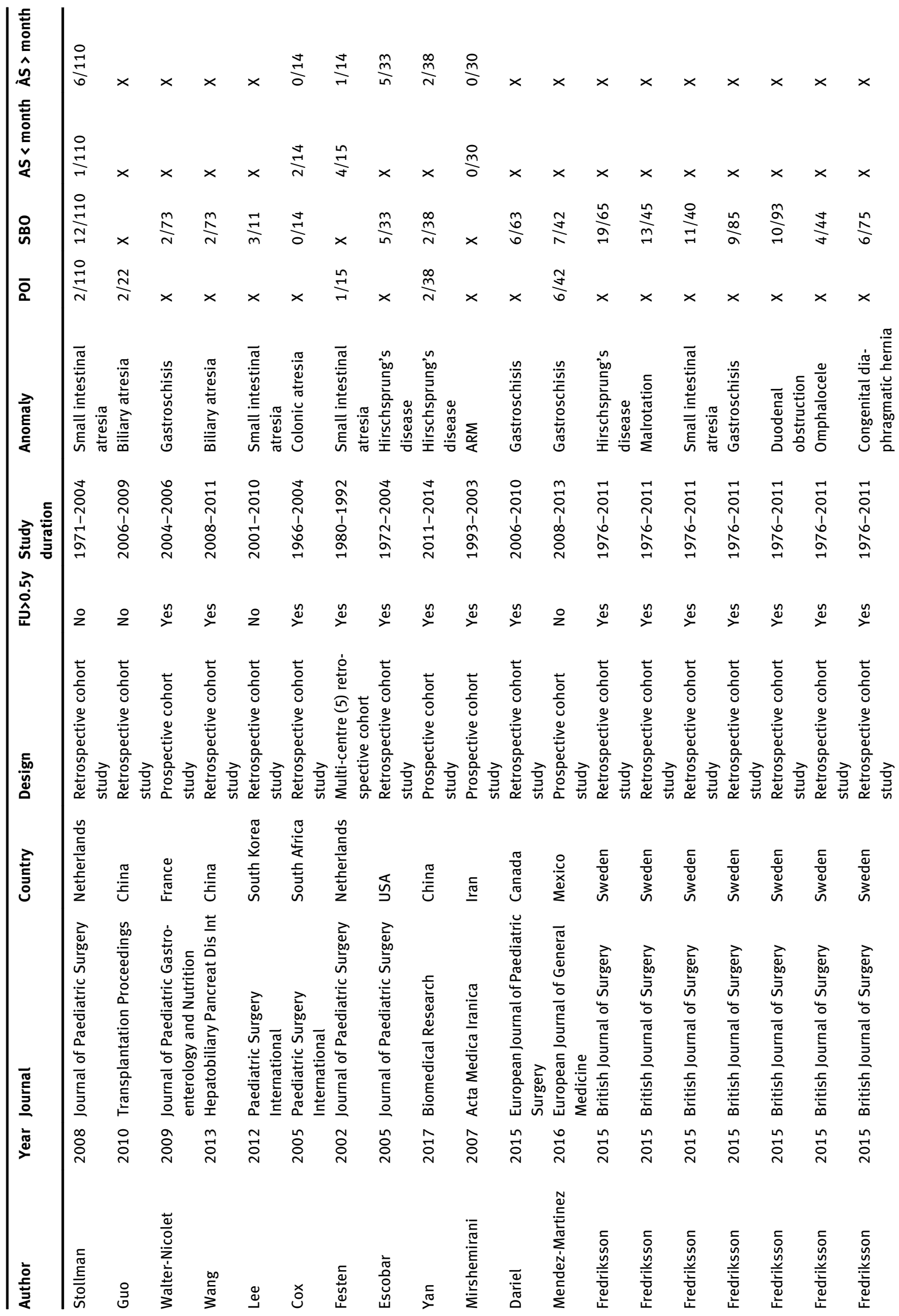




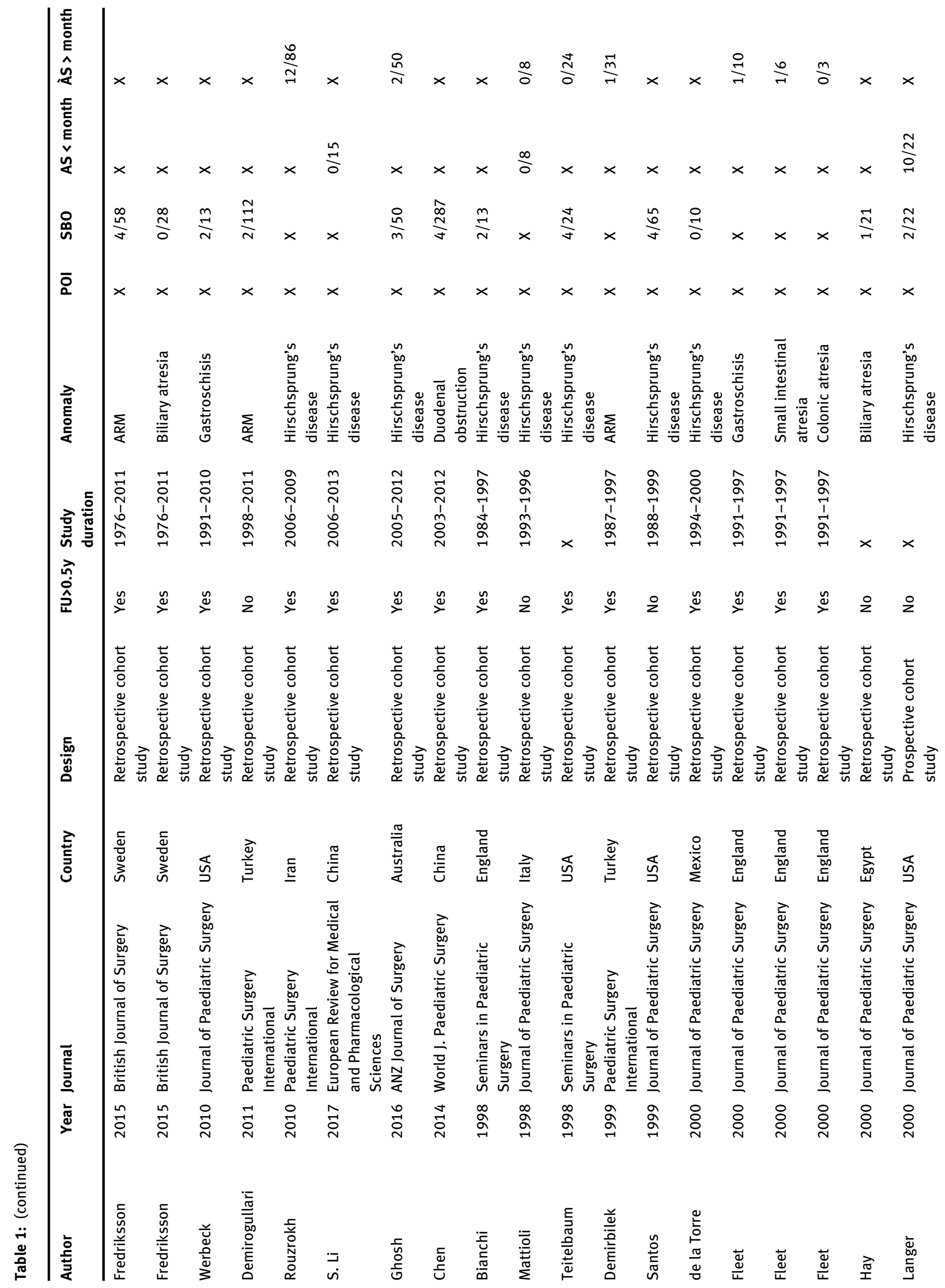




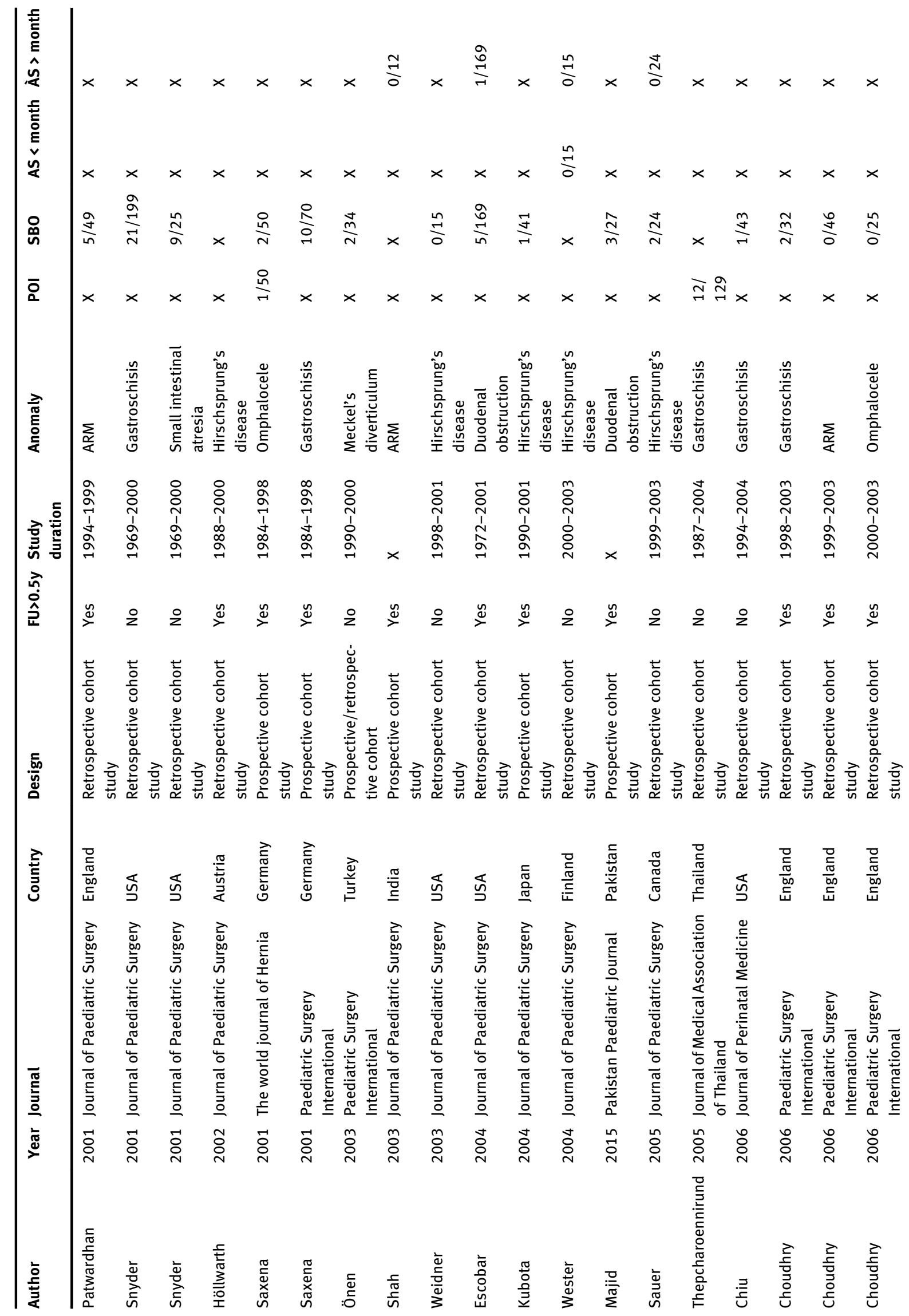




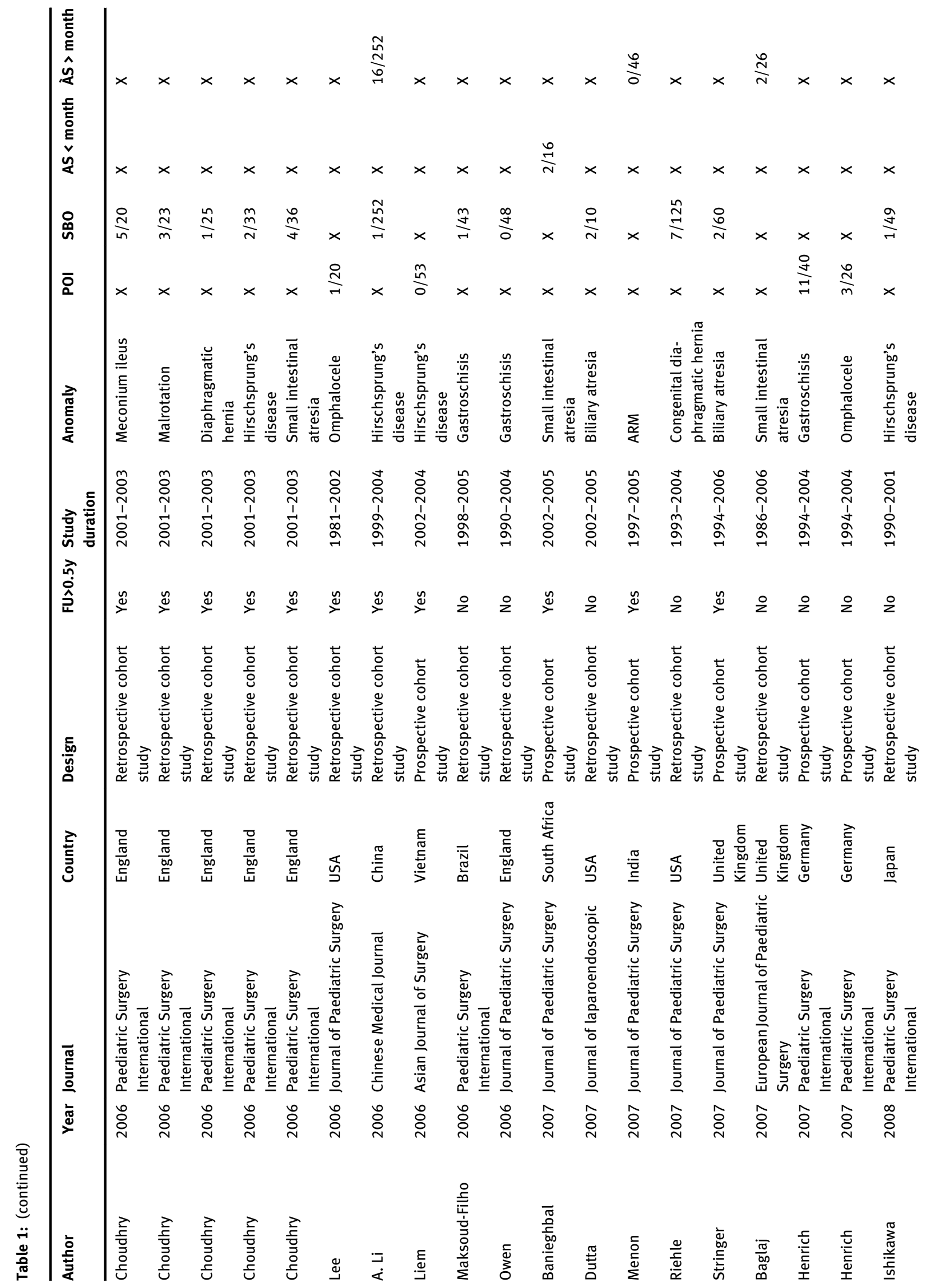




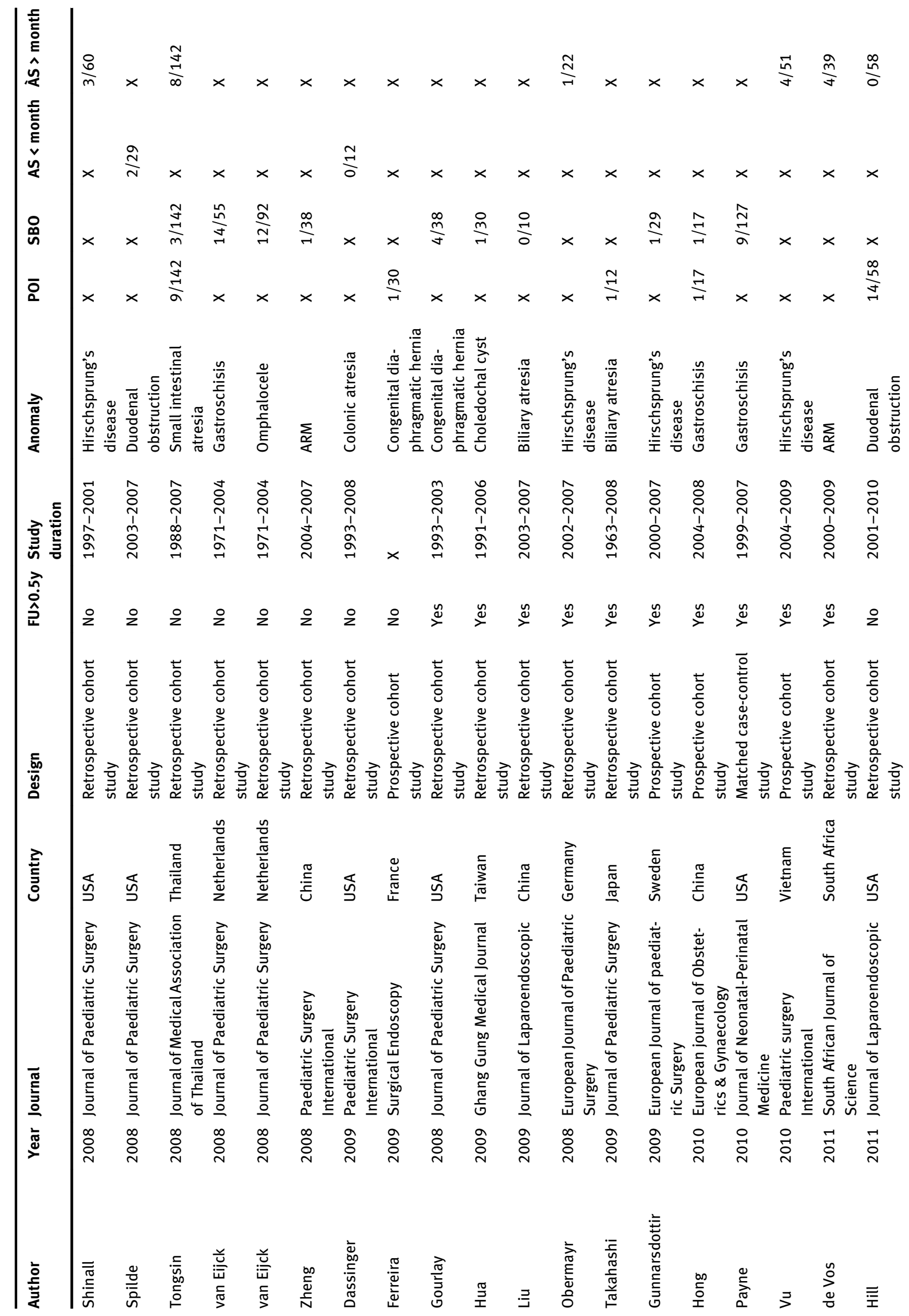




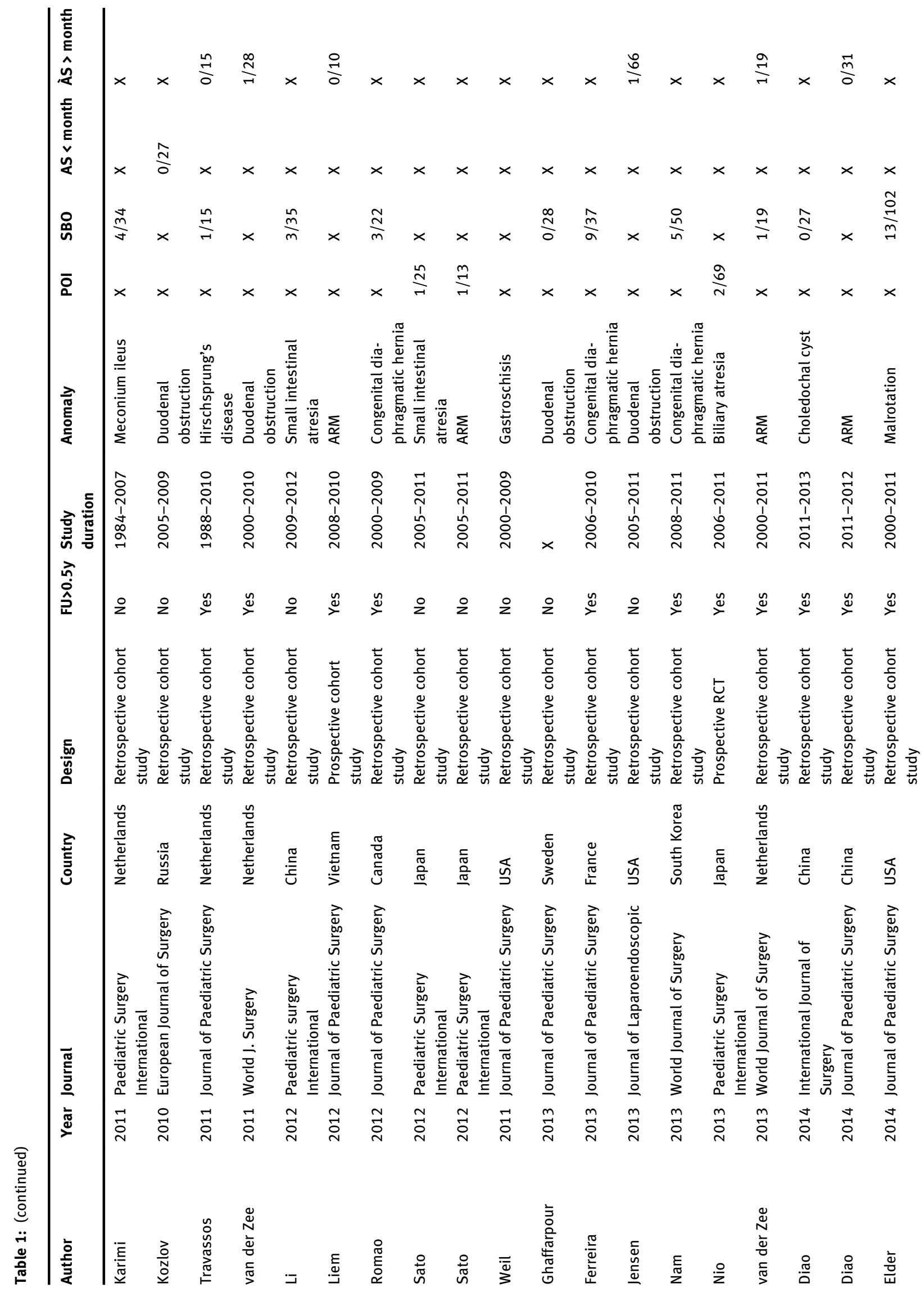




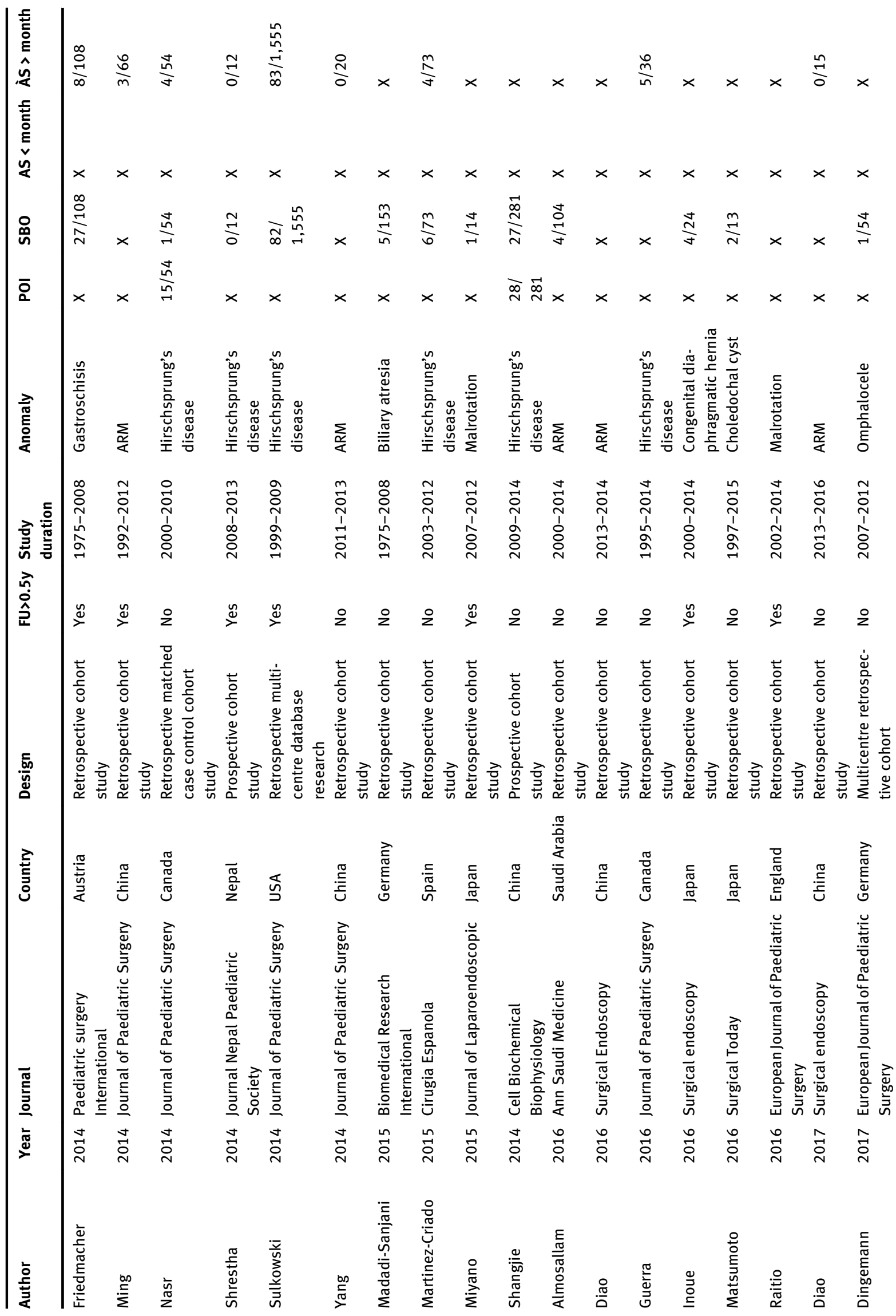




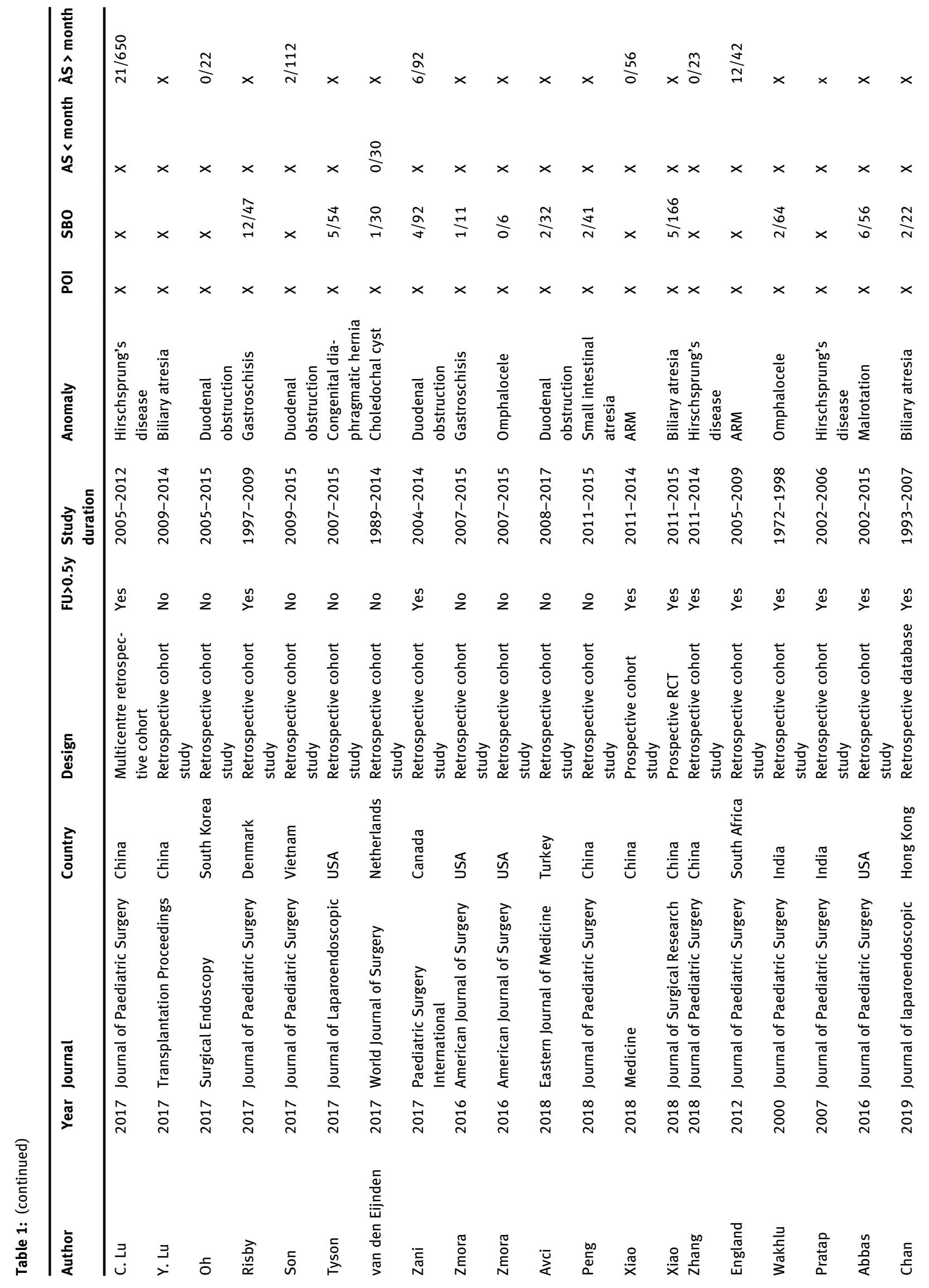




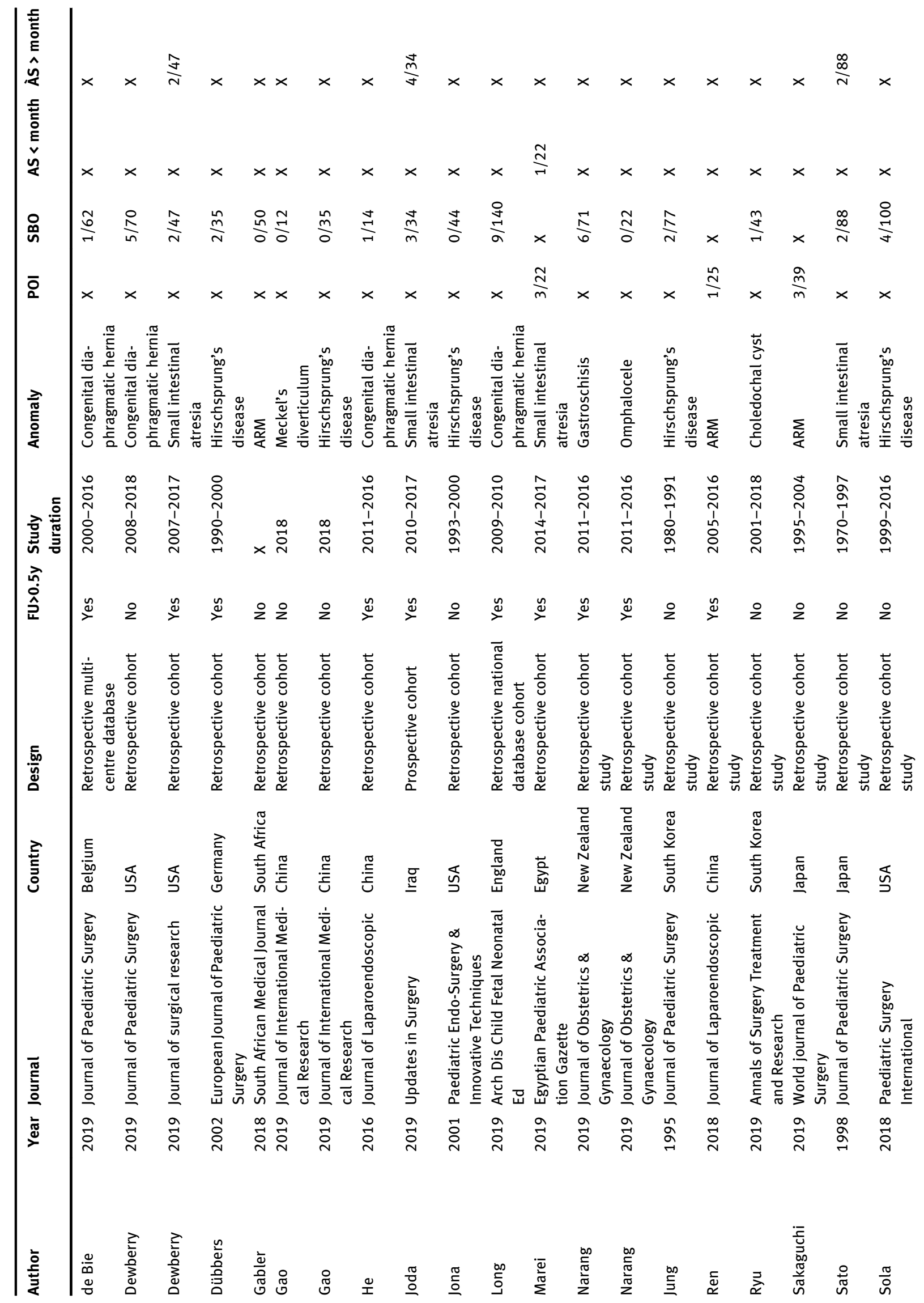




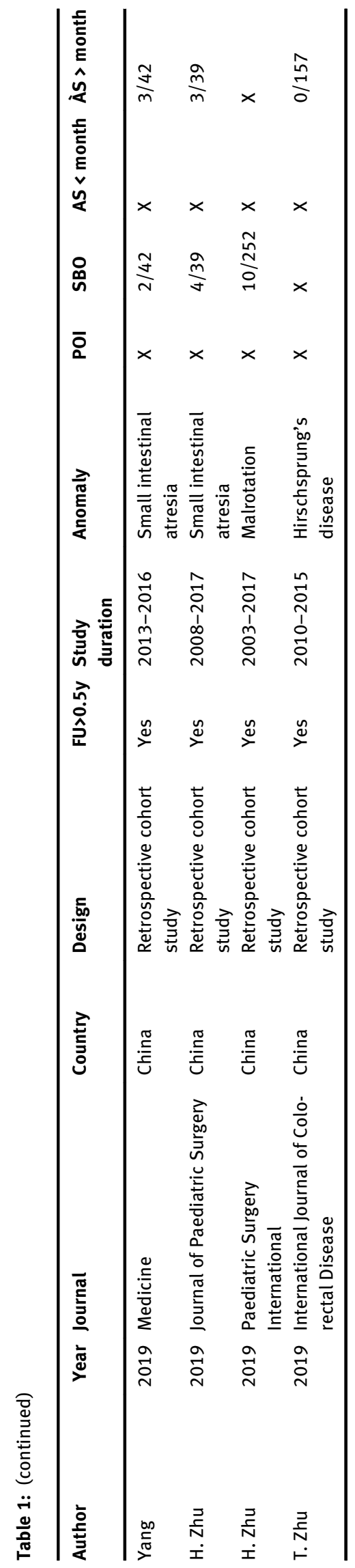

\section{Anastomotic stenosis}

Of the 14 studies reporting on anastomotic stenosis within one month of follow-up, 365 patients were included and 22 events of anastomotic stenosis occurred $[13,18,20,35,57$, 59, 64, 75, 78, 108, 109, 118, 129, 151].

The pooled proportion of total anastomotic stenosis within a month was 0.03 (95\%-CI: $0.01-0.10 ; I^{2}=81 \%$, $\mathrm{p}=0.02$ ). Diseases reported on were: small intestinal atresia $(n=8 / 163)$, Hirschsprung's disease $(n=10 / 60)$, duodenal obstruction $(n=2 / 56)$, anorectal malformations $(n=0 / 30)$, choledochal cyst $(n=0 / 30)$ and colonic atresia $(n=2 / 26)$.

In total, 40 studies reported on anastomotic stenosis after one month of follow-up entailing 4,468 patients and 214 events of anastomotic stenosis occurred [12, 18, 22, 23, $25,32-36,38,40,44,49,55,62,66,68,73,75-78,81,83,84$, 95, 99, 102, 104, 105, 107, 109, 111, 113, 115, 116, 119, 120, 122, $129,130,132-135,142,156,158,159,161]$. Length of follow up was at least half a year in 29 (73\%) of the studies.

The pooled proportion of anastomotic stenosis was 0.04 (95\%-CI: $\left.0.03-0.06 ; I^{2}=59 \%, p=0.30\right)$. Separate proportions were calculated for the following conditions: Hirschsprung's disease 0.04 (95\%-CI: 0.03-0.07; $\mathrm{n}=162$ / 3,238; $I^{2}=70 \% ; \mathrm{p}=0.11$ ); small intestinal atresia 0.06 (95\%-CI: 0.04-0.08; $\mathrm{n}=32 / 548 ; I^{2}=0 \% ; \mathrm{p}=0.77$ ); duodenal obstruction 0.02 (95\%-CI: $0.01-0.04 ; \mathrm{n}=11 / 547 ; I^{2}=42 \%$; $\mathrm{p}=0.29$ ); gastroschisis 0.08 (95\%-CI: 0.04-0.14; $\mathrm{n}=9 / 118$; $\left.I^{2}=0 \% ; \mathrm{p}=0.77\right)$. Colonic atresia $(\mathrm{n}=17)$ is included in the overall proportion but did not meet the criteria for separate statistical analysis (Figure 4).

\section{Discussion}

This systematic review pooled the reported proportions on different types of ileus following abdominal surgery for birth defects in infants. These proportions can be seen as an approximation of the incidences of these complications. According to our reported approximation, these incidences were $7 \%$ for paralytic ileus, $6 \%$ for adhesive small bowel obstruction, 3\% for anastomotic stenosis within one month after surgery and $4 \%$ after one month. Within the different birth defects there is a large variation in the occurrence and the spread of these forms of ileus. Although risk factor identification is beyond the scope of this review, the available literature gives some suggestions why these diseases seem to be more at risk.

Out of all diseases paralytic ileus was most common in gastroschisis patients (14\%). In these patients, a defect of the abdominal wall leads to extrusion of abdominal content 
Table 2: Assessment risk of bias.

\begin{tabular}{|c|c|c|c|c|c|c|}
\hline \multirow[t]{2}{*}{ Author } & \multirow[t]{2}{*}{ Year } & \multirow{2}{*}{$\begin{array}{l}\text { Jadad } \\
\text { Score } \\
(0-5)\end{array}$} & \multicolumn{4}{|c|}{ New Ottawa scale (NOS) } \\
\hline & & & $\begin{array}{r}\text { Selection } \\
\left(0-4^{*}\right)\end{array}$ & $\begin{array}{r}\text { Comparability } \\
\left(0-2^{*}\right)\end{array}$ & $\begin{array}{r}\text { Outcome } \\
\left(0-3^{*}\right)\end{array}$ & $\begin{array}{l}\text { Total } \\
(0-9)\end{array}$ \\
\hline Stollman & 2008 & & $\star \star \star$ & * & $\star \star \star$ & 7 \\
\hline Guo & 2010 & & $\star \star \star \star ~$ & * & ** & 6 \\
\hline Walter-Nicolet & 2009 & & $\star \star \star *$ & ** & 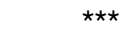 & 8 \\
\hline Wang & 2013 & & $\star \star \star$ & * & $\star \star \star$ & 7 \\
\hline Lee & 2012 & & $\star \star \star$ & * & $\star \star$ & 6 \\
\hline Cox & 2005 & & $\star \star \star ~$ & - & $\star \star \star$ & 6 \\
\hline Festen & 2002 & & ** & ** & $\star \star$ & 6 \\
\hline Escobar & 2005 & & $\star \star \star$ & - & $\star \star \star$ & 6 \\
\hline Yan & 2017 & & 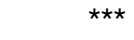 & ** & 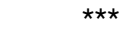 & 8 \\
\hline Mirshemirani & 2007 & & 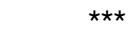 & - & 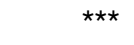 & 6 \\
\hline Dariel & 2015 & & $\star \star \star$ & * & $\star \star \star$ & 7 \\
\hline Mendez-Martinez & 2016 & & $\star \star \star$ & * & $\star \star$ & 6 \\
\hline Fredriksson & 2015 & & $\star \star \star *$ & - & $\star \star \star *$ & 6 \\
\hline Werbeck & 2010 & & ** & - & ** & 4 \\
\hline Demirogullari & 2011 & & $\star \star$ & - & ** & 4 \\
\hline Rouzrokh & 2010 & & $\star * \star$ & * & $* * \star$ & 7 \\
\hline S. Li & 2017 & & $\star \star$ & * & $\star \star \star$ & 6 \\
\hline Ghosh & 2016 & & ** & * & $\star \star \star *$ & 6 \\
\hline Chen & 2014 & & $\star \star \star$ & $\star \star$ & $\star \star \star$ & 8 \\
\hline Bianchi & 1998 & & $\star \star \star \star$ & - & $\star \star \star$ & 6 \\
\hline Mattioli & 1998 & & ** & - & $\star \star$ & 4 \\
\hline Teitelbaum & 1998 & & $\star \star$ & - & $\star \star \star$ & 5 \\
\hline Demirbilek & 1999 & & $\star \star \star$ & - & $\star \star \star$ & 6 \\
\hline Santos & 1999 & & $\star * \star$ & - & $* * \star$ & 6 \\
\hline de la Torre & 2000 & & $\star \star \star$ & - & $\star \star \star$ & 6 \\
\hline Fleet & 2000 & & $\star \star$ & * & 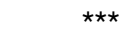 & 6 \\
\hline Hay & 2000 & & $\star \star \star *$ & - & $\star \star \star *$ & 6 \\
\hline Langer & 2000 & & $\star \star \star$ & * & $\star \star$ & 6 \\
\hline Patwardhan & 2001 & & $\star \star \star$ & * & $\star \star$ & 6 \\
\hline Snyder & 2001 & & $\star \star \star$ & $\star \star$ & $\star \star$ & 7 \\
\hline Höllwarth & 2002 & & $\star \star \star$ & - & $\star \star \star *$ & 6 \\
\hline Saxena & 2001 & & $\star \star \star \star$ & * & $\star \star \star$ & 7 \\
\hline Önen & 2003 & & $\star \star \star$ & - & $\star \star$ & 5 \\
\hline Shah & 2003 & & $\star \star$ & - & $\star * \star$ & 5 \\
\hline Weidner & 2003 & & $\star \star$ & $\star *$ & $\star \star$ & 6 \\
\hline Escobar & 2004 & & $\star \star \star *$ & * & $\star \star$ & 6 \\
\hline Kubota & 2004 & & ** & * & $\star \star$ & 5 \\
\hline Wester & 2004 & & $\star \star \star$ & - & $\star \star$ & 5 \\
\hline Majid & 2015 & & 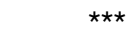 & ** & $\star \star \star \star ~$ & 8 \\
\hline Sauer & 2005 & & ** & ** & $\star \star$ & 6 \\
\hline Thepcharoennirund & 2005 & & ** & - & $\star \star$ & 4 \\
\hline Chiu & 2006 & & $\star \star \star$ & * & $\star \star$ & 6 \\
\hline Choudhry & 2006 & & $\star \star \star$ & - & $\star \star \star$ & 6 \\
\hline Lee & 2006 & & ** & * & $\star \star \star \star ~$ & 6 \\
\hline A. Li & 2006 & & $\star * \star$ & * & $\star \star \star *$ & 7 \\
\hline Liem & 2006 & & $\star \star$ & - & $\star \star$ & 4 \\
\hline Maksoud-Filho & 2006 & & $\star \star \star$ & - & $\star \star$ & 5 \\
\hline Owen & 2006 & & $\star \star$ & ** & $\star \star$ & 6 \\
\hline Banieghbal & 2007 & & $\star \star$ & - & $* \star \star$ & 5 \\
\hline Dutta & 2007 & & ** & - & $\star \star$ & 4 \\
\hline Menon & 2007 & & $\star \star$ & - & $\star \star \star$ & 5 \\
\hline Riehle & 2007 & & $\star \star \star *$ & - & $\star *$ & 5 \\
\hline Stringer & 2007 & & $\star \star \star \star$ & * & $\star \star$ & 6 \\
\hline Baglaj & 2007 & & $\star \star$ & * & $\star \star$ & 5 \\
\hline
\end{tabular}


Table 2: (continued)

\begin{tabular}{|c|c|c|c|c|c|c|}
\hline \multirow[t]{2}{*}{ Author } & \multirow[t]{2}{*}{ Year } & \multirow{2}{*}{$\begin{array}{l}\text { Jadad } \\
\text { Score } \\
(0-5)\end{array}$} & \multicolumn{4}{|c|}{ New Ottawa scale (NOS) } \\
\hline & & & $\begin{array}{r}\text { Selection } \\
\left(0-4^{*}\right)\end{array}$ & $\begin{array}{r}\text { Comparability } \\
\left(0-2^{*}\right)\end{array}$ & $\begin{array}{r}\text { Outcome } \\
\left(0-3^{*}\right)\end{array}$ & $\begin{array}{l}\text { Total } \\
(0-9)\end{array}$ \\
\hline Henrich & 2007 & & $\star \star \star$ & - & * & 4 \\
\hline Ishikawa & 2008 & & $\star \star \star \star ~$ & - & ** & 5 \\
\hline Shinall & 2008 & & $\star \star \star *$ & ** & ** & 7 \\
\hline Spilde & 2008 & & $\star \star \star$ & * & $\star \star$ & 6 \\
\hline Tongsin & 2008 & & $\star \star \star$ & * & $\star \star$ & 6 \\
\hline van Eijck & 2008 & & $\star \star \star$ & - & $\star \star$ & 5 \\
\hline Zheng & 2008 & & 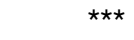 & - & $\star \star \star \star ~$ & 6 \\
\hline Dassinger & 2009 & & $\star \star \star \star ~$ & - & ** & 5 \\
\hline Ferreira & 2009 & & ** & - & ** & 4 \\
\hline Gourlay & 2008 & & 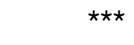 & - & 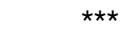 & 6 \\
\hline Hua & 2009 & & 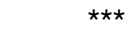 & * & $\star \star \star$ & 7 \\
\hline Liu & 2009 & & $\star \star \star \star ~$ & * & 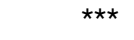 & 7 \\
\hline Obermayr & 2008 & & $\star \star \star$ & * & $\star \star \star \star$ & 7 \\
\hline Takahashi & 2009 & & ** & - & $\star \star \star \star$ & 5 \\
\hline Gunnarsdottir & 2009 & & $\star \star \star \star$ & ** & $\star \star \star$ & 8 \\
\hline Hong & 2010 & & ** & * & ** & 5 \\
\hline Payne & 2010 & & $\star \star \star \star *$ & * & $\star \star \star$ & 8 \\
\hline $\mathrm{Vu}$ & 2010 & & $\star \star \star \star ~$ & * & $\star \star \star$ & 7 \\
\hline de Vos & 2011 & & $\star \star \star$ & - & $\star \star \star \star$ & 6 \\
\hline Hill & 2011 & & $\star \star \star *$ & - & $\star \star$ & 5 \\
\hline Karimi & 2011 & & $\star \star \star$ & - & $\star \star$ & 5 \\
\hline Kozlov & 2010 & & 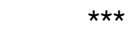 & $\star \star$ & $\star \star *$ & 7 \\
\hline Travassos & 2011 & & $\star \star \star$ & $\star \star$ & $\star \star \star$ & 8 \\
\hline van der Zee & 2011 & & $\star \star \star \star$ & - & $\star \star \star \star$ & 6 \\
\hline $\mathrm{Li}$ & 2012 & & $\star \star \star *$ & - & $\star \star$ & 5 \\
\hline Liem & 2012 & & ** & * & $\star \star$ & 5 \\
\hline Romao & 2012 & & $\star \star$ & * & 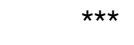 & 6 \\
\hline Sato & 2012 & & $\star \star \star$ & * & $\star \star$ & 6 \\
\hline Weil & 2011 & & $\star \star *$ & * & $\star \star \star *$ & 7 \\
\hline Ghaffarpour & 2013 & & $\star \star \star$ & * & $\star \star$ & 6 \\
\hline Ferreira & 2013 & & $\star \star \star$ & - & $\star \star \star *$ & 6 \\
\hline Jensen & 2013 & & $\star \star \star *$ & ** & $\star \star$ & 7 \\
\hline Nam & 2013 & & $\star \star \star$ & ** & $\star \star \star *$ & 8 \\
\hline Nio & 2013 & 2 & - & - & - & - \\
\hline van der Zee & 2013 & & $\star \star$ & - & $\star \star \star$ & 5 \\
\hline Diao & 2014 & & ** & * & $\star \star \star \star$ & 6 \\
\hline Elder & 2014 & & $\star \star \star$ & - & $\star \star \star \star$ & 6 \\
\hline Friedmacher & 2014 & & $\star \star \star$ & ** & 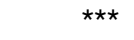 & 8 \\
\hline Ming & 2014 & & $\star \star \star \star ~$ & * & $\star \star \star \star$ & 7 \\
\hline Nasr & 2014 & & $\star \star \star \star$ & * & $\star \star \star \star$ & 7 \\
\hline Shrestha & 2014 & & $\star \star \star$ & - & $\star \star \star *$ & 6 \\
\hline Sulkowski & 2014 & & $\star \star \star *$ & ** & $* \star *$ & 8 \\
\hline Yang & 2014 & & $\star \star$ & - & $\star \star$ & 4 \\
\hline Madadi-Sanjani & 2015 & & $\star \star \star \star ~$ & ** & ** & 7 \\
\hline Martinez-Criado & 2015 & & $\star \star \star *$ & ** & $\star \star$ & 7 \\
\hline Miyano & 2015 & & $\star \star \star$ & - & 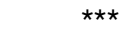 & 6 \\
\hline Shangjie & 2014 & & $\star \star \star$ & * & $\star \star$ & 6 \\
\hline Almosallam & 2016 & & $\star \star \star$ & - & $\star \star$ & 5 \\
\hline Diao & 2016 & & $\star \star$ & - & $\star \star \star \star$ & 5 \\
\hline Guerra & 2016 & & $\star \star \star$ & * & $\star \star$ & 6 \\
\hline Inoue & 2016 & & $\star \star$ & - & $\star \star \star \star$ & 5 \\
\hline Matsumoto & 2016 & & $\star \star \star$ & * & $\star \star$ & 6 \\
\hline Raitio & 2016 & & $\star \star * *$ & - & $\star \star \star$ & 6 \\
\hline
\end{tabular}


Table 2: (continued)

\begin{tabular}{|c|c|c|c|c|c|c|}
\hline \multirow[t]{2}{*}{ Author } & \multirow[t]{2}{*}{ Year } & \multirow{2}{*}{$\begin{array}{l}\text { Jadad } \\
\text { Score } \\
(0-5)\end{array}$} & \multicolumn{4}{|c|}{ New Ottawa scale (NOS) } \\
\hline & & & $\begin{array}{r}\text { Selection } \\
\left(0-4^{\star}\right)\end{array}$ & $\begin{array}{r}\text { Comparability } \\
\left(0-2^{*}\right)\end{array}$ & $\begin{array}{r}\text { Outcome } \\
\left(0-3^{*}\right)\end{array}$ & $\begin{array}{l}\text { Total } \\
(0-9)\end{array}$ \\
\hline Diao & 2017 & & ** & - & $\star \star$ & 4 \\
\hline Dingemann & 2017 & & $\star \star \star$ & * & $\star \star \star$ & 7 \\
\hline C. Lu & 2017 & & $\star \star \star$ & - & $\star \star \star ~$ & 6 \\
\hline Y. Lu & 2017 & & $\star \star \star$ & * & $\star \star \star$ & 7 \\
\hline $\mathrm{Oh}$ & 2017 & & $\star \star \star$ & ** & $\star \star$ & 7 \\
\hline Risby & 2017 & & $\star \star \star$ & - & $\star \star$ & 5 \\
\hline Son & 2017 & & 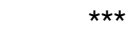 & ** & ** & 7 \\
\hline Tyson & 2017 & & $\star \star \star ~$ & * & $\star \star$ & 6 \\
\hline van den Eijnden & 2017 & & $\star \star \star$ & * & 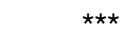 & 7 \\
\hline Zani & 2017 & & $\star \star \star$ & * & $\star \star \star ~$ & 7 \\
\hline Zmora & 2016 & & $\star \star \star$ & - & $\star \star$ & 5 \\
\hline Avci & 2018 & & $\star \star \star$ & * & ** & 6 \\
\hline Peng & 2018 & & $\star \star \star$ & * & $\star *$ & 6 \\
\hline Xiao & 2018 & 3 & - & - & - & - \\
\hline Zhang & 2018 & & $\star \star$ & * & $\star \star \star ~$ & 6 \\
\hline England & 2012 & & $\star \star \star \star$ & - & $\star \star \star$ & 6 \\
\hline Wakhlu & 2000 & & $\star \star \star *$ & - & $\star \star$ & 5 \\
\hline Pratap & 2007 & & $\star \star \star$ & - & 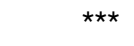 & 6 \\
\hline Abbas & 2016 & & $\star \star \star \star$ & * & $\star \star \star$ & 7 \\
\hline Chan & 2019 & & $\star \star$ & * & $\star \star$ & 5 \\
\hline de Bie & 2019 & & $\star \star$ & * & $\star \star \star *$ & 6 \\
\hline Dewberry & 2019 & & $\star \star \star$ & ** & $\star \star$ & 7 \\
\hline Dübbers & 2002 & & $\star \star \star$ & - & $\star \star$ & 5 \\
\hline Gabler & 2018 & & $\star \star \star$ & - & ** & 5 \\
\hline Gao & 2019 & & $\star *$ & - & $\star \star$ & 4 \\
\hline $\mathrm{He}$ & 2016 & & $\star \star \star \star \star$ & * & $\star \star \star *$ & 8 \\
\hline Joda & 2019 & & $\star \star \star *$ & ** & $\star \star \star ~$ & 8 \\
\hline Jona & 2001 & & $\star \star$ & - & $\star \star$ & 4 \\
\hline Long & 2019 & & 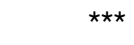 & $\star \star$ & $\star \star \star$ & 8 \\
\hline Marei & 2019 & & $\star \star$ & * & $\star \star \star$ & 6 \\
\hline Narang & 2019 & & $\star \star$ & * & 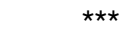 & 6 \\
\hline Jung & 1995 & & $\star \star$ & - & * & 3 \\
\hline Ren & 2018 & & $\star \star$ & * & $\star \star$ & 5 \\
\hline Ryu & 2019 & & $\star \star \star$ & * & $\star *$ & 6 \\
\hline Sakaguchi & 2019 & & $\star \star \star$ & * & ** & 6 \\
\hline Sato & 1998 & & $\star \star \star$ & - & ** & 5 \\
\hline Sola & 2018 & & $\star \star \star \star$ & * & $\star *$ & 6 \\
\hline Yang & 2019 & & $\star \star \star \star$ & * & $\star \star$ & 6 \\
\hline H. Zhu & 2019 & & $\star \star$ & $\star \star$ & $\star \star \star$ & 7 \\
\hline T. Zhu & 2019 & & $\star \star \star \star$ & * & $\star \star$ & 6 \\
\hline
\end{tabular}

antenatally. Postnatally, this content is reduced intra-abdominally either by primary closure or temporally use of silo and delayed closure. During both procedures the intestine is manipulated severely, which is known to increase the incidence and duration of paralytic ileus in adults [4].

Adhesions, which cause SBO, have long been accepted as a partly inevitable consequence of surgery. They occur as part of the natural healing process. It is hard to define the clinical significance of adhesions, since most are asymptomatic, but when they lead to small bowel obstruction, they can be fatal with mortality rates in children between 2 and 15\% [121, 162, 163]. Recently duration of surgery and staged procedures have been identified as risk factors for SBO [37, 162, 164].

Our reported pooled incidence of $6 \%$ is comparable to most recent large $(n \geq 100)$ individual cohort studies reporting on abdominal surgery in infants. These studies report an incidence of SBO between 6 and $10 \%$ [37, 165]. It is important to acknowledge that this review entails an aggregated 


\section{Pooled proportion of Paralytic lleus (PI)}

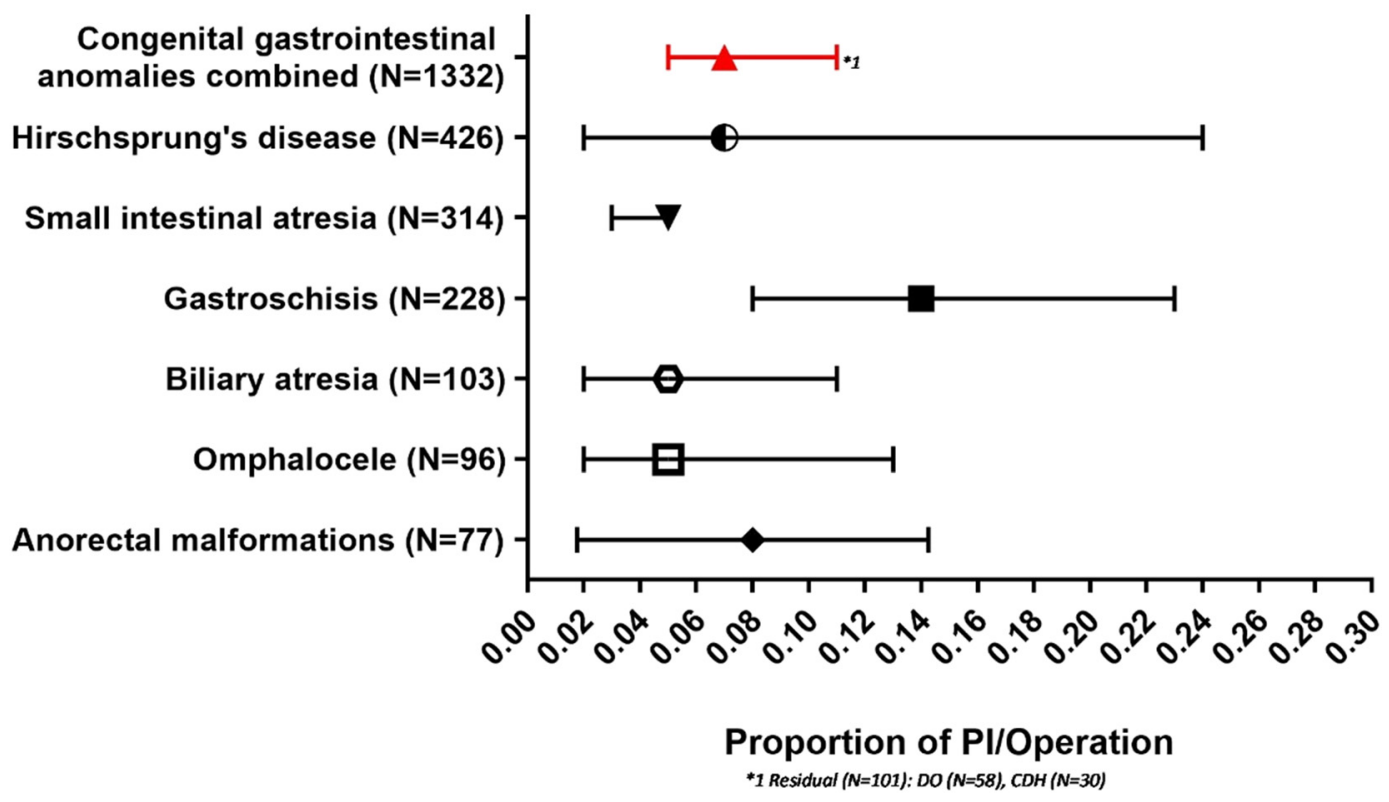

Figure 2: Pooled proportion of paralytic ileus.

\section{Pooled proportion of Small Bowel Obstruction (SBO)}

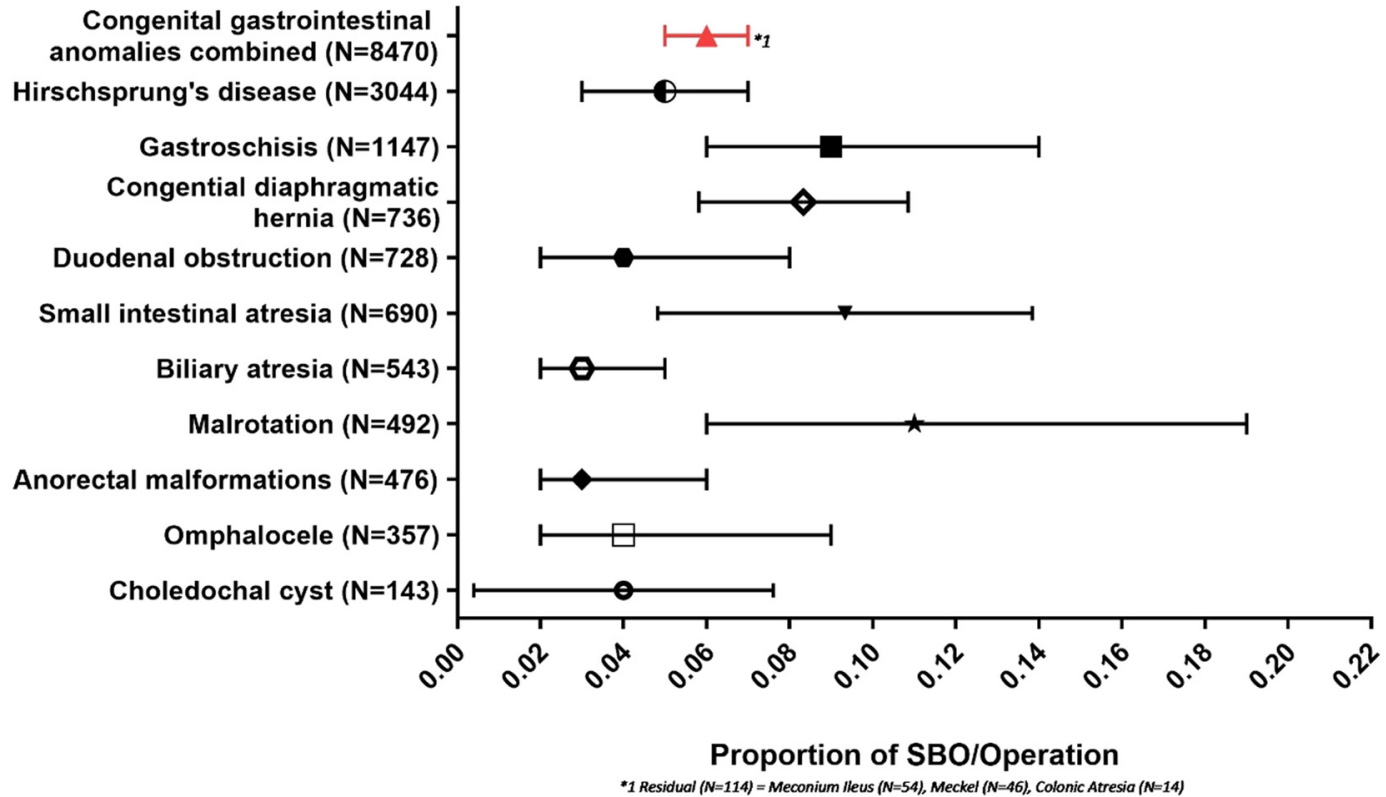

Figure 3: Pooled proportion of small bowel obstruction.

incidence for birth defects only. Acquired diseases such as necrotizing enterocolitis, which seems to be at high risk with a reported incidence of SBO between 25 and 64\%, are therefore not included [37, 162].

We found that patients with a malrotation, small intestinal atresia or gastroschisis were relatively most at risk of SBO. This is in concordance with previous studies [37, 121, 162-165].

We divided anastomotic stenosis into two groups based on reported occurrence within or after one month of surgery since early onset is suggested to be caused by technical error or tissue oedema, whereas a delayed onset 


\section{Pooled proportion of Anastomotic Stenosis (AS) after 1 month}

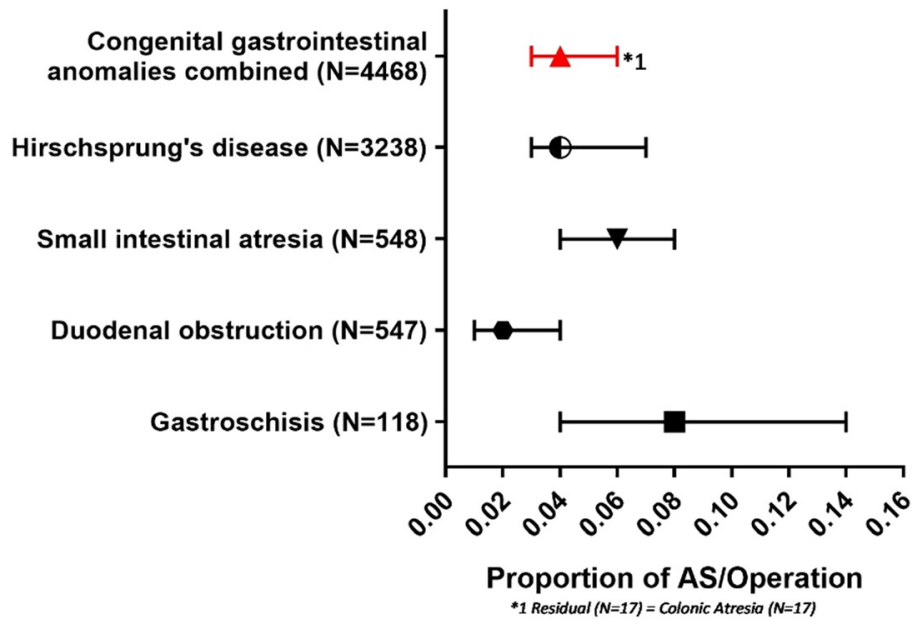

Figure 4: Pooled proportion of anastomotic stenosis after 1 month. and stricture formation is related to chronic inflammation in time leading to anastomotic scarring [166].

Early onset of an anastomotic stenosis is not widely reported and might even be overlooked in the infantile cohort. This review shows that early stenosis does occur and should be considered when conducting research into post-operative complications in the infantile cohort. Technical factors, such as suture reportion speed or mode of suturing, of influence during anastomotic creation should be evaluated to identify risk factors.

Gastroschisis, and to a smaller extent intestinal atresias, were most at risk for late onset anastomotic stenosis. The process of anastomotic healing is to a great extent unclear. Most research has focussed on surgical innovations and techniques without the results leading to a conclusive resolution. Future research in the pathobiology at the cellular level might bring clarification on this matter [166].

This study has its limitations. Because of the variety in study designs and reported outcomes we were not able to look into risk factors which could have lowered heterogeneity. Although it must be noted that, by stratifying for birth defect, some outcomes had moderate to low heterogeneity. Another limitation was that because certain birth defects such as gastroschisis only occur in neonates, our stratification might have resulted in differences in mean age when comparing birth defects. This age difference could be an important reason why certain birth defects are more at risk of certain form of ileus. However, it is not the aim of this review to compare different birth defects but rather report an incidence for each individually. Thus, we believe that this age difference will not hinder the message of our review. If we had only included neonates in this review important birth defects, such as Hirschsprung's disease, diagnosed beyond the neonatal period would have been excluded. Furthermore, it has to be stated that our results are based on retrospective cohorts available in the literature most of which did not have ileus as a primary outcome. This has undoubtedly increased the chances of occurrence of forms of bias such as selection, publication and reporting bias. Our risk of bias assessment showed most articles to have only fair quality mostly caused by the retrospective, observational nature of most included studies. Moreover, most studies did not have a strict definition of complications possibly resulting in observer bias. Lastly, only $57 \%$ of the included articles had a follow-up of at least half a year. Many other articles were unclear about the length of follow up. This lack of long-term follow-up might result in an underestimate of the real incidence of SBO and anastomotic stenosis. SBOs, for instance mostly arise within a year after surgery however episodes are reported 28 years after the initial laparotomy [17, 37, 121, 162-165]. Although these limitations might have influenced the pooled analyses, at this moment the presented data is the best available approximation of these complications in this cohort.

\section{Conclusion}

This review is the first to aggregate the known literature in order to approximate the incidence of different forms of ileus for each abdominal birth defect. We showed these complications are common and the distribution differs between birth defects. Knowing which birth defects are most 
at risk might aid clinicians in taking prompt action when an ileus is suspected. Future research should focus on the identification of risk factors and preventative measures. The incidences provided by this review can be used as a starting point for sample size calculations.

Acknowledgments: We would like to thank Shaffy Roell for the support with the development of the illustrations.

Research funding: None declared.

Author contributions: All authors have accepted responsibility for the entire content of this manuscript and approved its submission.

Competing interests: Authors state no conflict of interest. Informed consent: Not applicable.

Ethical approval: Not applicable.

\section{References}

1. Catania VD, Lauriti G, Pierro A, Zani A. Open versus laparoscopic approach for intestinal malrotation in infants and children: a systematic review and meta-analysis. Pediatr Surg Int 2016;32: 1157-64.

2. Schneuer FJ, Adams SE, Bentley JP, Holland AJ, Huckel Schneider C, White L, et al. A population-based comparison of the postoperative outcomes of open and laparoscopic appendicectomy in children. Med J Aust 2018;209:80-5.

3. Langer JC, Rollins MD, Levitt M, Gosain A, Torre L, Kapur RP, et al. Guidelines for the management of postoperative obstructive symptoms in children with Hirschsprung disease. Pediatr Surg Int 2017;33:523-6.

4. Bragg D, El-Sharkawy AM, Psaltis E, Maxwell-Armstrong CA, Lobo DN. Postoperative ileus: recent developments in pathophysiology and management. Clin Nutr 2015;34:367-76.

5. Venara A, Neunlist M, Slim K, Barbieux J, Colas PA, Hamy A, et al. Postoperative ileus: pathophysiology, incidence, and prevention. J Visc Surg 2016;153:439-46.

6. Shamseer L, Moher D, Clarke M, Ghersi D, Liberati A, Petticrew M, et al. Preferred reporting items for systematic review and metaanalysis protocols (PRISMA-P) 2015: elaboration and explanation. BMJ 2015;350:g7647.

7. Wells GA, Shea B, Higgins JP, Sterne J, Tugwell P, Reeves BC. Checklists of methodological issues for review authors to consider when including non-randomized studies in systematic reviews. Res Synth Methods 2013;4:63-77.

8. Jadad AR, Moore RA, Carroll D, Jenkinson C, Reynolds DJ, Gavaghan DJ, et al. Assessing the quality of reports of randomized clinical trials: is blinding necessary? Contr Clin Trials 1996;17:1-12.

9. Higgins JPT, Thompson SG, Deeks JJ, Altman DG. Measuring inconsistency in meta-analyses. BMJ 2003;327:557-60.

10. Almosallam OI, Aseeri A, Shanafey SA. Outcome of loop versus divided colostomy in the management of anorectal malformations. Ann Saudi Med 2016;36:352-5.

11. Avci V, Bilici S, Düz E, Beger B, Değer İ. Congenital duodenal obstruction: ten-year results of a tertiary center. E J Med 2018;23: 191-4.
12. Baglaj M, Carachi R, Lawther S. Multiple atresia of the small intestine: a 20-year review. Eur J Pediatr Surg 2008;18:13-8.

13. Banieghbal B, Beale PG. Minimal access approach to jejunal atresia. J Pediatr Surg 2007;42:1362-4.

14. Bianchi A. One-stage neonatal reconstruction without stoma for Hirschsprung's disease. Semin Pediatr Surg 1998;7:170-3.

15. Chen QJ, Gao ZG, Tou JF, Qian YZ, Li MJ, Xiong QX, et al. Congenital duodenal obstruction in neonates: a decade's experience from one center. World J Pediatr 2014;10:238-44.

16. Chiu B, Lopoo J, Hoover JD, Almond PS, Arensman R, Madonna $M B$. Closing arguments for gastroschisis: management with silo reduction. J Perinat Med 2006;34:243-5.

17. Choudhry MS, Grant HW. Small bowel obstruction due to adhesions following neonatal laparotomy. Pediatr Surg Int 2006;22:729-32.

18. Cox SG, Numanoglu A, Millar AJ, Rode H. Colonic atresia: spectrum of presentation and pitfalls in management. A review of 14 cases. Pediatr Surg Int 2005;21:813-8.

19. Dariel A, Poocharoen W, Silva N, Pleasants H, Gerstle J. Secondary plastic closure of gastroschisis is associated with a lower incidence of mechanical ventilation. Eur J Pediatr Surg 2014;25. https://doi.org/10.1055/s-0034-1395487.

20. Dassinger M, Jackson R, Smith S. Management of colonic atresia with primary resection and anastomosis. Pediatr Surg Int 2009; 25:579-82.

21. De la Torre L, Ortega A. Transanal versus open endorectal pullthrough for Hirschsprung's disease. J Pediatr Surg 2000;35: 1630-2.

22. De Vos C, Arnold M, Sidler D, Moore SW. A comparison of laparoscopic-assisted (LAARP) and posterior sagittal (PSARP) anorectoplasty in the outcome of intermediate and high anorectal malformations. S Afr J Surg 2011;49:39-43.

23. Demirbilek S, Atayurt HF. Anal transposition without colostomy: functional results and complications. Pediatr Surg Int 1999;15: 221-3.

24. Demirogullari B, Yilmaz Y, Yildiz G, Ozen I, Karabulut R, Turkyilmaz Z, et al. Ostomy complicatlons in patients with anorectal malformations. Pediatr Surg Int 2011;27:1075-8.

25. Diao M, Li L, Guan KP, Zhang Z, Cheng W. A novel laparoscopic technique for anorectal malformation with low recto-bulbar fistulae. Surg Endosc 2017;31:4326-30.

26. Diao M, Li L, Li Q, Ye M, Cheng W. Challenges and strategies for single-incision laparoscopic Roux-en-Y hepaticojejunostomy in managing giant choledochal cysts. Int J Surg 2014;12:412-7.

27. Diao M, Li L, Ye M, Cheng W. Single-incision laparoscopicassisted anorectoplasty using conventional instruments for children with anorectal malformations and rectourethral or rectovesical fistula. J Pediatr Surg 2014;49:1689-94.

28. Diao M, Li L, Ye M, Guan KP, Wei YD, Cheng W. Congenital anomaly rectified at birth: one-stage single-incision laparoscopic-assisted anorectoplasty for newborns with anorectal malformations and recto-urethral fistula. Surg Endosc 2016;30:5156-64.

29. Dingemann C, Dietrich J, Zeidler J, Blaser J, Gosemann JH, Lacher $M$, et al. Surgical management of congenital abdominal wall defects in Germany: a population-based study and comparison with literature reports. Eur J Pediatr Surg 2017;27:516-25.

30. Dutta S, Woo R, Albanese CT. Minimal access portoenterostomy: advantages and disadvantages of standard laparoscopic and robotic techniques. J Laparoendosc Adv Surg Tech A 2007;17: 258-64. 
31. Elder CT, Metzger R, Arrington C, Rollins M, Scaife E. The role of screening and prophylactic surgery for malrotation in heterotaxy patients. J Pediatr Surg 2014;49:1746-8.

32. England RJ, Warren SL, Bezuidenhout L, Numanoglu A, Millar AJ. Laparoscopic repair of anorectal malformations at the red cross war Memorial Children's Hospital: taking stock. J Pediatr Surg 2012;47:565-70.

33. Escobar MA, Grosfeld JL, West KW, Scherer LR, Rouse TM, Engum SA, et al. Long-term outcomes in total colonic aganglionosis: a 32-year experience. J Pediatr Surg 2005;40: 955-61.

34. Escobar MA, Ladd AP, Grosfeld JL, West KW, Rescorla FJ, Scherer LR 3rd, et al. Duodenal atresia and stenosis: long-term follow-up over 30 years. J Pediatr Surg 2004;39:867-71.

35. Festen S, Brevoord JC, Goldhoorn GA, Festen C, Hazebroek FW, van Heurn LW, et al. Excellent long-term outcome for survivors of apple peel atresia. J Pediatr Surg 2002;37:61-5.

36. Fleet MS, de la Hunt MN. Intestinal atresia with gastroschisis: a selective approach to management. J Pediatr Surg 2000;35: 1323-5.

37. Fredriksson F, Christofferson RH, Lilja HE. Adhesive small bowel obstruction after laparotomy during infancy. Br J Surg 2016;103: 284-9.

38. Friedmacher F, Hock A, Castellani C, Avian A, Hollwarth ME. Gastroschisis-related complications requiring further surgical interventions. Pediatr Surg Int 2014;30:615-20.

39. Ghaffarpour N, Svensson PJ, Svenningsson A, Wester T, Mesas Burgos C. Supraumbilical incision with U-u umbilicoplasty for congenital duodenal atresia: the Stockholm experience. J Pediatr Surg 2013;48:1981-5.

40. Ghosh DN, Liu Y, Cass DT, Soundappan SSV. Transition zone pullthrough in Hirschsprung's disease: a tertiary hospital experience. ANZ J Surg 2017;87:780-3.

41. Gomes Ferreira C, Kuhn P, Lacreuse I, Kasleas C, Philippe P, Podevin $\mathrm{G}$, et al. Congenital diaphragmatic hernia: an evaluation of risk factors for failure of thoracoscopic primary repair in neonates. J Pediatr Surg 2013;48:488-95.

42. Gomes Ferreira C, Reinberg O, Becmeur F, Allal H, De Lagausie P, Lardy $\mathrm{H}$, et al. Neonatal minimally invasive surgery for congenital diaphragmatic hernias: a multicenter study using thoracoscopy or laparoscopy. Surg Endosc 2009;23:1650-9.

43. Gourlay DM, Cassidy LD, Sato TT, Lal DR, Arca MJ. Beyond feasibility: a comparison of newborns undergoing thoracoscopic and open repair of congenital diaphragmatic hernias. J Pediatr Surg 2009;44:1702-7.

44. Guerra J, Wayne C, Musambe T, Nasr A. Laparoscopic-assisted transanal pull-through (LATP) versus complete transanal pullthrough (CTP) in the surgical management of Hirschsprung's disease. J Pediatr Surg 2016;51:770-4.

45. Gunnarsdottir A, Larsson LT, Arnbjornsson E. Transanal endorectal vs. Duhamel pull-through for Hirschsprung's disease. Eur J Pediatr Surg 2010;20:242-6.

46. Guo CB, Li YC, Zhang MM, Yan LN, Pu CL, Kang Q, et al. Early postoperative care of liver transplantation for infants with biliary atresia during pediatric intensive care unit stay. Transplant Proc 2010;42:1750-4

47. Hay SA, Soliman HE, Sherif HM, Abdelrahman AH, Kabesh AA, Hamza AF. Neonatal jaundice: the role of laparoscopy. J Pediatr Surg 2000;35:1706-9.
48. Henrich K, Huemmer HP, Reingruber B, Weber PG. Gastroschisis and omphalocele: treatments and long-term outcomes. Pediatr Surg Int 2008;24:167-73.

49. Hill S, Koontz CS, Langness SM, Wulkan ML. Laparoscopic versus open repair of congenital duodenal obstruction in infants. J Laparoendosc Adv Surg Tech A 2011;21:961-3.

50. Hollwarth ME, Rivosecchi M, Schleef J, Deluggi S, Fasching G, Ceriati $\mathrm{E}$, et al. The role of transanal endorectal pull-through in the treatment of Hirschsprung's disease - a multicenter experience. Pediatr Surg Int 2002;18:344-8.

51. Hong L, Wu YM, Yan ZL, Chen S, Wang J. Modified silo techniquean easy and effective method to improve the survival rate of neonates with gastroschisis in Shanghai. Eur J Obstet Gynecol Reprod Biol 2010;148:31-4.

52. Hua MC, Chao HC, Lien R, Lai JY, Lai MW, Kong MS. The different clinical and liver pathological characteristics between the newborns and infants with choledochal cysts. Chang Gung Med J 2009;32:198-203.

53. Inoue M, Uchida K, Otake K, Nagano Y, Mori K, Hashimoto K, et al. Thoracoscopic repair of congenital diaphragmatic hernia with countermeasures against reported complications for safe outcomes comparable to laparotomy. Surg Endosc 2016;30: 1014-9.

54. Ishikawa N, Kubota A, Kawahara H, Hasegawa T, Okuyama H, Uehara S, et al. Transanal mucosectomy for endorectal pullthrough in Hirschsprung's disease: comparison of abdominal, extraanal and transanal approaches. Pediatr Surg Int 2008;24 $1127-9$.

55. Jensen AR, Short SS, Anselmo DM, Torres MB, Frykman PK, Shin $\mathrm{CE}$, et al. Laparoscopic versus open treatment of congenital duodenal obstruction: multicenter short-term outcomes analysis. J Laparoendosc Adv Surg Tech A 2013;23:876-80.

56. Karimi A, Gorter RR, Sleeboom C, Kneepkens CM, Heij HA. Issues in the management of simple and complex meconium ileus. Pediatr Surg Int 2011;27:963-8.

57. Kozlov Y, Novogilov V, Yurkov P, Podkamenev A, Weber I, Sirkin N. Keyhole approach for repair of congenital duodenal obstruction. Eur J Pediatr Surg 2011;21:124-7.

58. Kubota A, Kawahara H, Okuyama H, Oue T, Tazuke Y, Okada A. Clinical outcome of laparoscopically assisted endorectal pullthrough in Hirschsprung's disease: comparison of abdominal and perineal approaches. J Pediatr Surg 2004;39:1835-7.

59. Langer JC, Seifert M, Minkes RK. One-stage Soave pull-through for Hirschsprung's disease: a comparison of the transanal and open approaches. J Pediatr Surg 2000;35:820-2.

60. Lee SH, Cho YH, Kim HY, Park JH, Byun SY. Clinical experience of complex jejunal atresia. Pediatr Surg Int 2012;28:1079-83.

61. Lee SL, Beyer TD, Kim SS, Waldhausen JH, Healey PJ, Sawin RS, et al. Initial nonoperative management and delayed closure for treatment of giant omphaloceles. J Pediatr Surg 2006;41: 1846-9.

62. Li AW, Zhang WT, Li FH, Cui XH, Duan XS. A new modification of transanal Soave pull-through procedure for Hirschsprung's disease. Chin Med J (Engl) 2006;119:37-42.

63. Li B, Chen WB, Wang SQ, Liu SL, Li L. Laparoscopy-assisted surgery for neonatal intestinal atresia and stenosis: a report of 35 cases. Pediatr Surg Int 2012;28:1225-8.

64. Li SX, Zhang HW, Cao H, Zou HX, Yin YY, Sui F, et al. Clinical effects of ascending colon patching ileorectal heart-shaped 
anastomosis on total colonic aganglionosis. Eur Rev Med Pharmacol Sci 2017;21(4 Suppl):90-4.

65. Liem NT, Hau BD. Primary laparoscopic endorectal colon pullthrough for Hirschsprung's disease: early results of 61 cases. Asian J Surg 2006;29:173-5.

66. Liem NT, Quynh TA. Laparoscopic rectal pull-through for persistent cloaca: an easier approach for a complex anomaly. J Pediatr Surg 2012;47:815-8.

67. Liu SL, Li L, Cheng W, Hou WY, Huang LM, Wang WY, et al. Laparoscopic hepatojejunostomy for biliary atresia. J Laparoendosc Adv Surg Tech A 2009;19(1 Suppl):S31-5.

68. Lu C, Hou G, Liu C, Geng Q, Xu X, Zhang J, et al. Single-stage transanal endorectal pull-through procedure for correction of Hirschsprung disease in neonates and nonneonates: a multicenter study. J Pediatr Surg 2017;52:1102-7.

69. Lu Y, Xia Q, Yang Y, Wan P, Hou J, Wang Y, et al. Effect of preoperative growth status on clinical outcomes after living-donor liver transplantation in infants. Transplant Proc 2017;49:1848-54.

70. Madadi-Sanjani O, Carl N, Longerich T, Petersen C, Andruszkow $\mathrm{JH}$. Inguinal hernias represent the most frequent surgical complication after Kasai in biliary atresia infants. BioMed Res Int 2015;2015:383791.

71. Majid F, Sheikh AH, Ramzan M, Siddique M. Duodenal obstruction in neonates: management and outcome. Pak Paediatr J 2015;39:93-7.

72. Maksoud-Filho JG, Tannuri U, da Silva MM, Maksoud JG. The outcome of newborns with abdominal wall defects according to the method of abdominal closure: the experience of a single center. Pediatr Surg Int 2006;22:503-7.

73. Martinez-Criado Y, Cabrera R, Moya MJ, Valladares JC, LopezAlonso M, De Agustin Asensio JC. Results of transanal endorrectal descent in Hirschprung's disease. Cir Esp 2015;93:561-6.

74. Matsumoto M, Urushihara N, Fukumoto K, Yamoto M, Miyake H, Nakajima H. Laparoscopic management for prenatally diagnosed choledochal cysts. Surg Today 2016;46:1410-4.

75. Mattioli G, Buffa P, Martinelli M, Ivani G, Jasonni V. All mechanical low rectal anastomosis in children. J Pediatr Surg 1998;33: 503-6.

76. Menon P, Rao KL. Primary anorectoplasty in females with common anorectal malformations without colostomy. J Pediatr Surg 2007;42:1103-6.

77. Ming AX, Li L, Diao M, Wang HB, Liu Y, Ye M, et al. Long term outcomes of laparoscopic-assisted anorectoplasty: a comparison study with posterior sagittal anorectoplasty. J Pediatr Surg 2014;49:560-3.

78. Mirshemirani AR, Kouranlou J, Rouzrokh M, Sadeghian M, Khaleghnezhad A. Primary posterior sagittal anorectoplasty without colostomy in neonates with high imperforate anus. Acta Med Iran 2007;45:121-5.

79. Miyano G, Fukuzawa H, Morita K, Kaneshiro M, Miyake H, Nouso $\mathrm{H}$, et al. Laparoscopic repair of malrotation: what are the indications in neonates and children? J Laparoendosc Adv Surg Tech A 2015;25:155-8.

80. Nam SH, Cho MJ, Kim DY, Kim SC. Shifting from laparotomy to thoracoscopic repair of congenital diaphragmatic hernia in neonates: early experience. World J Surg 2013;37:2711-6.

81. Nasr A, Haricharan RN, Gamarnik J, Langer JC. Transanal pullthrough for Hirschsprung disease: matched case-control comparison of Soave and Swenson techniques. J Pediatr Surg 2014;49:774-6.
82. Nio M, Muraji T. Multicenter randomized trial of postoperative corticosteroid therapy for biliary atresia. Pediatr Surg Int 2013; 29:1091-5.

83. Obermayr F, Szavay P, Beschorner R, Fuchs J. Outcome of transanal endorectal pull-through in patients with hirschsprung's disease. Eur J Pediatr Surg 2009;19:220-3.

84. Oh C, Lee S, Lee SK, Seo JM. Laparoscopic duodenoduodenostomy with parallel anastomosis for duodenal atresia. Surg Endosc 2017;31:2406-10.

85. Onen A, Cigdem MK, Ozturk H, Otcu S, Dokucu Al. When to resect and when not to resect an asymptomatic Meckel's diverticulum: an ongoing challenge. Pediatr Surg Int 2003;19:57-61.

86. Owen A, Marven S, Jackson L, Antao B, Roberts J, Walker J, et al. Experience of bedside preformed silo staged reduction and closure for gastroschisis. J Pediatr Surg 2006;41:1830-5.

87. Patwardhan N, Kiely EM, Drake DP, Spitz L, Pierro A. Colostomy for anorectal anomalies: high incidence of complications. J Pediatr Surg 2001;36:795-8.

88. Payne NR. A cross-sectional, case-control follow-up of infants with gastroschisis. In: Gilmore L, editor. Journal of neonatalperinatal medicine; 2010. https://doi.org/10.3233/npm-20100117.

89. Peng Y, Zheng H, He Q, Wang Z, Zhang H, Chaudhari PB, et al. Is the Bishop-Koop procedure useful in severe jejunoileal atresia? J Pediatr Surg 2018;53:1914-7.

90. Pratap A, Gupta DK, Shakya VC, Adhikary S, Tiwari A, Shrestha P, et al. Analysis of problems, complications, avoidance and management with transanal pull-through for Hirschsprung disease. J Pediatr Surg 2007;42:1869-76.

91. Raitio A, Green PA, Fawkner-Corbett DW, Wilkinson DJ, Baillie CT. Malrotation: age-related differences in reoperation rate. Eur J Pediatr Surg 2016;26:34-7.

92. Riehle KJ, Magnuson DK, Waldhausen JH. Low recurrence rate after Gore-Tex/Marlex composite patch repair for posterolateral congenital diaphragmatic hernia. J Pediatr Surg 2007;42:1841-4.

93. Risby K, Husby S, Qvist N, Jakobsen MS. High mortality among children with gastroschisis after the neonatal period: a long-term follow-up study. J Pediatr Surg 2017;52:431-6.

94. Romao RL, Nasr A, Chiu PP, Langer JC. What is the best prosthetic material for patch repair of congenital diaphragmatic hernia? Comparison and meta-analysis of porcine small intestinal submucosa and polytetrafluoroethylene. J Pediatr Surg 2012;47: 1496-500.

95. Rouzrokh M, Khaleghnejad AT, Mohejerzadeh L, Heydari A, Molaei $\mathrm{H}$. What is the most common complication after one-stage transanal pull-through in infants with Hirschsprung's disease? Pediatr Surg Int 2010;26:967-70.

96. Méndez-Martinez S, García-Carrasco M, Mendoza-Pinto C. Surgical complications of simple and complex gastroschisis in newborn. Eur J gen med 2016.

97. Santos MC, Giacomantonio JM, Lau HY. Primary Swenson pullthrough compared with multiple-stage pull-through in the neonate. J Pediatr Surg 1999;34:1079-81.

98. Sato K, Uchida H, Tanaka Y, Takazawa S, Jimbo T, Deie K. Stapled intestinal anastomosis is a simple and reliable method for management of intestinal caliber discrepancy in children. Pediatr Surg Int 2012;28:893-8.

99. Sauer CJ, Langer JC, Wales PW. The versatility of the umbilical incision in the management of Hirschsprung's disease. J Pediatr Surg 2005;40:385-9. 
100. Saxena A, Willital GH. Omphalocele: clinical review and surgical experience using dura patch grafts. Hernia 2002;6:73-8.

101. Saxena AK, Hulskamp G, Schleef J, Schaarschmidt K, Harms E, Willital GH. Gastroschisis: a 15-year, single-center experience. Pediatr Surg Int 2002;18:420-4.

102. Shah AJ, Bhattacharjee N, Patel DN, Ganatra JR. Anal shift: preliminary results. J Pediatr Surg 2003;38:196-8.

103. Shangjie X, Xiaochun Z, Wenyi Y, Wuping G, Ying Z, Qiuming H, et al. TGF-beta1, Ghrelin, Neurexin, and Neuroligin are predictive biomarkers for postoperative prognosis of laparoscopic surgery in children with Hirschsprung disease. Cell Biochem Biophys 2015;71:1249-54.

104. Shinall MC, Jr., Koehler E, Shyr Y, Lovvorn HN 3rd. Comparing cost and complications of primary and staged surgical repair of neonatally diagnosed Hirschsprung's disease. J Pediatr Surg 2008;43:2220-5.

105. Shrestha MK, Sherchan M, Dhaubhadel BK, Basnet RB. Early experience with single-stage transanal endorectal pull through for rectosigmoid Hirschsprung's disease. J Nepal Paediatr Soc 2014;34:188-92.

106. Snyder CL, Miller KA, Sharp RJ, Murphy JP, Andrews WA, Holcomb GW 3rd, et al. Management of intestinal atresia in patients with gastroschisis. J Pediatr Surg 2001;36:1542-5.

107. Son TN, Kien HH. Laparoscopic versus open surgery in management of congenital duodenal obstruction in neonates: a single-center experience with 112 cases. J Pediatr Surg 2017;52: 1949-51.

108. Spilde TL, St Peter SD, Keckler SJ, Holcomb GW 3rd, Snyder CL, Ostlie DJ. Open vs laparoscopic repair of congenital duodenal obstructions: a concurrent series. J Pediatr Surg 2008;43:1002-5.

109. Stollman TH, de Blaauw I, Wijnen MH, van der Staak FH, Rieu PN, Draaisma JM, et al. Decreased mortality but increased morbidity in neonates with jejunoileal atresia; a study of 114 cases over a 34-year period. J Pediatr Surg 2009;44:217-21.

110. Stringer MD, Davison SM, Rajwal SR, McClean P. Kasai portoenterostomy: 12-year experience with a novel adjuvant therapy regimen. J Pediatr Surg 2007;42:1324-8.

111. Sulkowski JP, Cooper JN, Congeni A, Pearson EG, Nwomeh BC, Doolin EJ, et al. Single-stage versus multi-stage pull-through for Hirschsprung's disease: practice trends and outcomes in infants. J Pediatr Surg 2014;49:1619-25.

112. Takahashi Y, Matsuura T, Saeki I, Zaizen Y, Taguchi T. Excellent long-term outcome of hepaticojejunostomy for biliary atresia with a hilar cyst. J Pediatr Surg 2009;44:2312-5.

113. Teitelbaum DH, Coran AG. Primary pull-through in the newborn. Semin Pediatr Surg 1998;7:103-7.

114. Thepcharoennirund S. Primary fascial closure in 112 infants with gastroschisis. J Med Assoc Thai 2005;88:492-7.

115. Tongsin A, Anuntkosol M, Niramis R. Atresia of the jejunum and ileum: what is the difference? J Med Assoc Thai 2008;91(3 Suppl):S85-9.

116. Travassos DV, van der Zee DC. Is complete resection of the aganglionic bowel in extensive total aganglionosis up to the middle ileum always necessary? J Pediatr Surg 2011;46: 2054-9.

117. Tyson AF, Sola R Jr., Arnold MR, Cosper GH, Schulman AM. Thoracoscopic versus open congenital diaphragmatic hernia repair: single tertiary center review. J Laparoendosc Adv Surg Tech A 2017;27:1209-16.
118. van den Eijnden MHA, de Kleine RHJ, de Blaauw I, Peeters P, Koot BPG, Oomen MWN, et al. Choledochal malformation in children: lessons learned from a Dutch national study. World J Surg 2017; 41:2631-7.

119. van der Zee DC. Laparoscopic repair of duodenal atresia: revisited. World J Surg 2011;35:1781-4.

120. van der Zee DC, Dik P, Beek FJ. Laparoscopy-assisted anorectal pull-through in anorectal malformations: a reappraisal. World J Surg 2013;37:1934-9.

121. van Eijck FC, Wijnen RM, van Goor H. The incidence and morbidity of adhesions after treatment of neonates with gastroschisis and omphalocele: a 30-year review. J Pediatr Surg 2008;43:479-83.

122. Vu PA, Thien HH, Hiep PN. Transanal one-stage endorectal pullthrough for Hirschsprung disease: experiences with 51 newborn patients. Pediatr Surg Int 2010;26:589-92.

123. Wakhlu A, Wakhlu AK. The management of exomphalos. J Pediatr Surg 2000;35:73-6.

124. Walter-Nicolet E, Rousseau V, Kieffer F, Fusaro F, Bourdaud N, Oucherif $\mathrm{S}$, et al. Neonatal outcome of gastroschisis is mainly influenced by nutritional management. J Pediatr Gastroenterol Nutr 2009;48:612-7.

125. Wang Q, Yan LN, Zhang MM, Wang WT, Zhao JC, Pu CL, et al. The pre-Kasai procedure in living donor liver transplantation for children with biliary atresia. Hepatobiliary Pancreat Dis Int 2013; 12:47-53.

126. Weidner BC, Waldhausen JH. Swenson revisited: a one-stage, transanal pull-through procedure for Hirschsprung's disease. I Pediatr Surg 2003;38:1208-11.

127. Weil BR, Leys CM, Rescorla FJ. The jury is still out: changes in gastroschisis management over the last decade are associated with both benefits and shortcomings. J Pediatr Surg 2012;47: 119-24.

128. Werbeck R, Koltai J. Umbilical cord as temporary coverage in gastroschisis. Eur J Pediatr Surg 2011;21:292-5.

129. Wester T, Rintala RJ. Early outcome of transanal endorectal pullthrough with a short muscle cuff during the neonatal period. I Pediatr Surg 2004;39:157-60.

130. Xiao H, Huang R, Chen L, Diao M, Cheng W, Li L, et al. The midterm outcomes of 1-stage versus 3-stage laparoscopicassisted anorectoplasty in anorectal malformations with rectoprostatic fistula and rectobulbar fistula: a retrospective cohort study. Medicine (Baltim) 2018;97: e11843.

131. Xiao H, Huang R, Chen L, Diao M, Li L. The application of a shorter loop in Kasai portoenterostomy reconstruction for Ohi type III biliary atresia: a prospective randomized controlled trial. J Surg Res 2018;232:492-6.

132. Yan J, Li S, Chang Y, Wang J, Li A. Comparison of transcolostomy single-incision laparoscopic and open procedures in secondary operations for Hirschsprung's disease. Biomed Res 2017;28: 3527-31.

133. Yang L, Tang ST, Li S, Aubdoollah TH, Cao GQ, Lei HY, et al. Two-stage laparoscopic approaches for high anorectal malformation: transumbilical colostomy and anorectoplasty. J Pediatr Surg 2014;49:1631-4.

134. Zani A, Yeh JB, King SK, Chiu PP, Wales PW. Duodenoduodenostomy or duodeno-jejunostomy for duodenal atresia: is one repair better than the other? Pediatr Surg Int 2017;33: 245-8. 
135. Zhang X, Cao GQ, Tang ST, Chang XP, Li S, Yang L, et al. Laparoscopic-assisted Duhamel procedure with ex-anal rectal transection for total colonic aganglionosis. J Pediatr Surg 2018; 53:531-6.

136. Zheng S, Xiao X, Huang Y. Single-stage correction of imperforate anus with a rectourethral or a rectovestibula fistula by semi-posterior sagittal anorectoplasty. Pediatr Surg Int 2008; 24:671-6.

137. Zmora O, Castle SL, Papillon S, Stein JE. The biological prosthesis is a viable option for abdominal wall reconstruction in pediatric high risk defects. Am J Surg 2017;214:479-82.

138. Abbas PI, Dickerson HA, Wesson DE. Evaluating a management strategy for malrotation in heterotaxy patients. J Pediatr Surg 2016;51:859-62.

139. Chan KWE, Lee KH, Wong HYV, Tsui SYB, Mou JWC, Tam YHP. Tenyear native liver survival rate after laparoscopic and open Kasai portoenterostomy for biliary atresia. J Laparoendosc Adv Surg Tech A 2019;29:121-5.

140. De Bie F, Suply E, Verbelen T, Vanstraelen S, Debeer A, Cross K, et al. Early surgical complications after congenital diaphragmatic hernia repair by thoracotomy vs. laparotomy: a bicentric comparison. J Pediatr Surg 2020:32005504. https://doi.org/10. 1016/j.jpedsurg.2019.12.020. https://www.sciencedirect.com/ science/article/pii/S0022346819309303?via\%3Dihub.

141. Dewberry L, Hilton S, Gien J, Liechty KW, Marwan Al. Flap repair in congenital diaphragmatic hernia leads to lower rates of recurrence. J Pediatr Surg 2019;54:2487-91.

142. Dewberry LC, Hilton SA, Vuille-Dit-Bille RN, Liechty KW. Is tapering enteroplasty an alternative to resection of dilated bowel in small intestinal atresia? J Surg Res 2020;246:1-5.

143. Dubbers M, Holschneider AM, Meier-Ruge W. Results of total and subtotal colon resections in children. Eur J Pediatr Surg 2003;13: 195-200.

144. Gabler TD, Loveland J, Theron A, Westgarth-Taylor C. Anorectal malformations and the impact of HIV on surgical outcome. S Afr Med J 2018;108. https://doi.org/10.7196/SAMJ.2018.v108i11.13168.

145. Gao R, Yang H, Li Y, Meng L, Li Y, Sun B, et al. Enhanced recovery after surgery in pediatric gastrointestinal surgery. J Int Med Res 2019;47:4815-26.

146. He QM, Zhong W, Zhang H, Li L, Wang Z, Tan Y, et al. Standardized indications to assist in the safe thoracoscopic repair of congenital diaphragmatic hernia in neonates. J Laparoendosc Adv Surg Tech A 2016;26:399-403.

147. Joda AE, Abdullah AF. Outcomes of end-to-side oblique anastomosis as a surgical technique for jejuno-ileal atresia. Updates Surg 2019;71:587-93.

148. Jona J. Personal experience with 50 laparoscopic procedures for Hirschsprung's disease in infants and children. Pediatr Endosurg Innovative Tech 2001;5:361-3.

149. Jung PM. Hirschsprung's disease: one surgeon's experience in one institution. J Pediatr Surg 1995;30:646-51.

150. Long AM, Bunch KJ, Knight M, Kurinczuk JJ, Losty PD. One-year outcomes of infants born with congenital diaphragmatic hernia: a national population cohort study. Arch Dis Child Fetal Neonatal Ed 2019;104:F643-7.

151. Marei MM, Abouelfadl MH, Rawwash AAE, Seleim HM, Mahmoud WM, Yassin TYM, et al. Early outcomes of tapering jejunoplasty by antimesenteric seromuscular stripping and mucosal inversion for proximal jejunal atresia. Egypt Pediatr Assoc Gaz 2019;67:4.

152. Narang A, Carlsen V, Long A, Battin M, Upadhyay V, Sadler L, et al. Anterior abdominal wall defects managed at a tertiary maternal-fetal medicine service in New Zealand: what counselling advice can we offer parents? Aust N Z J Obstet Gynaecol 2019;59:805-10.

153. Ren X, Xiao H, Li L, Diao M, Chen L, Zhou R, et al. Single-incision laparoscopic-assisted anorectoplasty versus three-port laparoscopy in treatment of persistent cloaca: a midterm followup. J Laparoendosc Adv Surg Tech A 2018;28:1540-7.

154. Ryu HS, Lee JY, Kim DY, Kim SC, Namgoong J-M. Minimallyinvasive neonatal surgery: laparoscopic excision of choledochal cysts in neonates. Ann Surg Treat Res 2019;97:21-6.

155. Sakaguchi T, Hamada Y, Shirai T, Hamada H, Shigeta Y, Nakamura $Y$, et al. Postoperative complications of umbilical loop colostomy for anorectal malformations in neonates compared with the conventional abdominal stoma: a non-randomized study. World J Plast Surg 2019;2: e000031.

156. Sato S, Nishijima E, Muraji T, Tsugawa C, Kimura K. Jejunoileal atresia: a 27-year experience. J Pediatr Surg 1998;33:1633-5.

157. Sola R, Jr., Poola AS, Memon R, Singh V, Hendrickson RJ, St Peter SD, et al. The relationship of eosinophilia with outcomes of Hirschsprung disease in children. Pediatr Surg Int 2019;35:425-9.

158. Yang S, Wang M, Shen C. Bowel plication in neonatal high jejunal atresia. Medicine (Baltim) 2019;98: e15459.

159. Zhu H, Gao R, Alganabi M, Dong K, Ganji N, Xiao X, et al. Longterm surgical outcomes of apple-peel atresia. J Pediatr Surg 2019;54:2503-8.

160. Zhu H, Zheng S, Alganabi M, Peng X, Dong K, Pierro A, et al. Reoperation after Ladd's procedure in the neonatal period. Pediatr Surg Int 2019;35:117-20.

161. Zhu T, Sun X, Wei M, Yi B, Zhao X, Wang W, et al. Optimal time for single-stage pull-through colectomy in infants with short-segment Hirschsprung disease. Int J Colorectal Dis 2019;34:255-9.

162. Young JY, Kim DS, Muratore CS, Kurkchubasche AG, Tracy TF Jr., Luks Fl. High incidence of postoperative bowel obstruction in newborns and infants. J Pediatr Surg 2007;42:962-5.

163. Lakshminarayanan B, Hughes-Thomas AO, Grant HW. Epidemiology of adhesions in infants and children following open surgery. Semin Pediatr Surg 2014;23:344-8.

164. Grant HW, Parker MC, Wilson MS, Menzies D, Sunderland G, Thompson JN, et al. Adhesions after abdominal surgery in children. J Pediatr Surg 2008;43:152-6.

165. Wilkins BM, Spitz L. Incidence of postoperative adhesion obstruction following neonatal laparotomy. Br J Surg 1986;73: 762-4.

166. Guyton KL, Hyman NH, Alverdy JC. Prevention of perioperative anastomotic healing complications: anastomotic stricture and anastomotic leak. Adv Surg 2016;50:129-41.

Supplementary Material: The online version of this article offers reviewer assessments as supplementary material (https://doi.org/ 10.1515/iss-2020-0042). 